\title{
THE BRAUER-WALL GROUP OF A COMMUTATIVE RING $\left({ }^{1}\right)$
}

BY

\author{
CHARLES SMALL
}

\begin{abstract}
Let $k$ be a commutative ring (with 1 ). We work with $k$-algebras with a grading mod 2, and with graded modules over such algebras. Using graded notions of tensor product, commutativity, and morphisms, we construct an abelian group BW $(k)$ whose elements are suitable equivalence classes of Azumaya $k$-algebras. The consruction generalizes, and is patterned on, the definition of the Brauer group $\mathrm{Br}(k)$ given by Auslander and Goldman. $\mathrm{Br}(k)$ is in fact a subgroup of $\mathrm{BW}(k)$, and we describe the quotient as a group of graded quadratic extensions of $k$.
\end{abstract}

Introduction. The subject of this paper is a functor, BW, from commutative rings to abelian groups. The Brauer group $\mathrm{Br}(k)$ introduced by Auslander and Goldman in [1] is a subgroup of the Brauer-Wall group BW $(k)$, and the construction of BW is patterned on the construction of $\mathrm{Br}$. The "enlarged" Brauer group was considered for fields by C. T. C. Wali in [9], and the structure theory of [9], presented in $\$ 5$ below, is an important tool in our work here. Also among the principal sources for this paper we call attention to the Tata notes [3] of H. Bass. Chapter III of [3] presents much of the basic material of [1]; Chapter II presents the "Morita theory" which, suitably translated into our graded context, is another important tool for us; and the construction of BW is outlined in Chapter IV.

The original motivation for enlarging the Brauer group, in [3] as in [9], came from the study of quadratic forms. Various inconveniences in this theory are eliminated if the Clifford algebra of a form is viewed as an element of BW $(k)$ rather than $\mathrm{Br}(k)$. The Brauer-Wall group is therefore a fundamental tool towards a general theory of quadratic forms (over an arbitrary commutative ring). This theme is developed in [3, Chapter V].

A second motivation comes from algebraic $K$-theory. Karoubi has shown (in [6] and a series of subsequent papers) that Clifford algebras play a dramatic unifying role among the various $K$-theories of topology. One hopes that they, and Azumaya algebras generally, may similarly provide the key to a more

Received by the editors July 7, 1970.

AMS 1970 subject classifications. Primary 13A20, 16A16; Secondary 13B05, 18F25.

Key words and phrases. Brauer group of a commutative ring, separable algebra, Azumaya algebra, graded algebra, Galois extension of commutative rings, quadratic extension of commutative rings.

( $\left.{ }^{1}\right)$ This paper contains part of the author's doctoral thesis, and it is a pleasure for him to acknowledge here his gratitude to Professor Hyman Bass of Columbia University for inspiration and patient guidance while it was being written. 
comprehensive formulation of the algebraic theory. Any such application will have to allow algebras with a grading mod 2-hence BW $(k)$.

A word about the organization of the paper. The first two sections contain the basic definitions. $\$ 3$ develops two long strings of lemmas, and the reader is advised to use this section only for reference as needed in later sections, at least on a first reading. $\$ \S 4$ and 5 describe the machinery used to construct the Brauer-Wall group, and BW $(k)$ is finally defined in $\$ 6$. The first step toward an effective computation of $\mathrm{BW}(k)$ is to describe the quotient $\mathrm{BW} / \mathrm{Br}$, and this is done in $\S 7$. This quotient group can, in fact, be described quite explicitly in terms of the arithmetic (group of units, Picard group) of $k$. This is not carried out here, however, and will be the subject of a subsequent paper.

Much of the material presented here was outlined, without proofs, in Chapter IV of [3]. While we refer to earlier chapters of [3] for some proofs, we give full details for results stated in [3] without proof.

All rings have $1 \neq 0$, and all homomorphisms preserve 1 . Module, unqualified, means left module except where right modules are explicitly specified, and all modules, right or left, are unital.

1. Graded rings, modules, and algebras. A ring, $A$, is graded if additively it is a direct sum $A=A_{0} \oplus A_{1}$ of subgroups which, with respect to multiplication, satisfy

$$
A_{i} A_{j} \subset A_{i+j} \quad(i, j \in Z / 2 Z) .
$$

(1.1) implies that $1_{A} \in A_{0}$, so that $A_{0}$ is a ring and $A_{1}$ is an $A_{0}$-module. We let $|A|$ denote the underlying ungraded ring. A homomorphism $f: A \rightarrow B$ of graded rings is a homomorphism $|A| \rightarrow|B|$ such that $f\left(A_{i}\right) \subset B_{i}$.

A graded $A$-module, $M$, is an $|A|$-module which is additively a direct sum $M=M_{0} \oplus M_{1}$ satisfying

$$
A_{i} M_{j} \subset M_{i+j} \quad(i, j \in Z / 2 Z) .
$$

$|M|$ denotes the underlying ungraded $|A|$-module.

If $X$ is an ungraded ring or module, $(X)$ denotes the same object with grading, concentrated in degree zero: $(X)_{0}=X$ and $(X)_{1}=0$. If $N$ is an ungraded $R$-module, $(N)$ is an $(R)$-module.

A direct sum $M=\coprod_{i \in I} M^{i}$ of graded $A$-modules is a graded $A$-module if we set $M_{j}=\coprod_{i \in I} M_{j}^{i}$. If $M^{i}=N$ for all $i \in I$, we write $N^{(I)}$ for $\coprod_{i \in I} M^{i}$.

If $X$ is a graded ring or module, $h X$ denotes $X_{0} \cup X_{1}$, the set of homogeneous elements. $h X$ is a union of two subgroups whose intersection is 0 , but it is not itself a subgroup. If $0 \neq x \in h X, x \in X_{i}$ for $i=0$ or $i=1$ but not both, and we write $\partial x=i$, the degree of $x$. For any $Y \subset h X,\langle Y\rangle$ will denote the subgroup of $X$ generated by $Y$. Thus $Y \subset h\langle Y\rangle$, with equality if and only if $Y \cap X_{0}$ and $Y \cap X_{1}$ are subgroups.

Let $M$ and $N$ be graded $A$-modules. We let $\operatorname{HOM}_{A}(M, N)$ denote the graded 
abelian group $\operatorname{HOM}_{A}(M, N)_{0} \oplus \mathrm{HOM}_{A}(M, N)_{1}$, defined by the requirements that $f \in \operatorname{HOM}_{A}(M, N)_{j}$ (that is, $f \in h \operatorname{HOM}_{A}(M, N)$ and $\left.\partial f=j\right)$ if and only if

(a) $f\left(M_{i}\right) \subset N_{i+j}$, and

(b) $f(a x)=-1^{(\partial a) j} a(f x) \quad(a \in h A, x \in M)$.

(In (1.3(a)) the indices are of course read in $Z / 2 Z$.) We let $\operatorname{Hom}_{A}(M, N)$ denote $\operatorname{HOM}_{A}(M, N)_{0}$.

(1.3(b)), which can be written $f(a x)=-1^{\partial a \cdot \partial f} a(f x)$, is the first instance of a phenomenon which will recur quite often: The presence of a sign depending upon the degrees of certain homogeneous elements. We will usually simplify the notation by writing $-1^{x y}$ for $-1^{\partial x \cdot \partial y}$, and $-1^{(x y)}$ for $-1^{\partial(x y)}=-1^{\partial x+\partial y}$, even going so far as to write $-1^{x+y}$ for the latter.

If $f \in h \operatorname{HOM}_{A}(M, N)$ and $g \in h \operatorname{HOM}_{A}(N, X)$ then $g f \in h \operatorname{HOM}_{A}(M, X)$ and $\partial(g f)=\partial g+\partial f$. We will write $\operatorname{END}_{A}(M)\left(\operatorname{resp} . \operatorname{End}_{A}(M)\right)$ for $\operatorname{HOM}_{A}(M, M)$ (resp. $\operatorname{Hom}_{A}(M, M)$ ).

If $X$ is a graded ring or module, a subgroup $\Omega$ is called graded if

$$
\Omega=\left(\Omega \cap X_{0}\right) \oplus\left(\Omega \cap X_{1}\right),
$$

or equivalently, if $\Omega$ contains the images of the projections $\Omega \rightarrow X_{i}$. By a submodule, or an ideal (left, right, or two-sided), of $X$, we will always mean a graded subgroup $\Omega$ such that $|\Omega|$ is a submodule (or an ideal, of the appropriate type) of $|X|$. If we wish to speak of "possibly nongraded submodules (or ideals)" we have to speak of submodules (or ideals) of $|X|$. We insist on graded subgroups so that the quotient $X / \Omega$ will also be graded.

Let $A$ be a graded ring. $A$-MOD will denote the category whose objects are all graded $A$-modules, with morphisms $\mathrm{HOM}_{A}$, and $A$-Mod will denote the category with the same objects but only $\mathrm{Hom}_{A}$ as morphisms. $A$-Mod is abelian, but $A$-MOD is not; for example the kernel of a nonhomogeneous morphism can easily fail to be a submodule.

$\tau$ will denote the degree-shifting function: If $f \in \operatorname{HOM}_{A}(M, N),[\tau M]_{i}=M_{i+1}$ $(i \in Z / 2 Z)$, and " $\tau f=f$ " in the obvious sense: $\tau f \in \operatorname{HOM}_{A}(\tau M, \tau N)$, and if $f$ is homogeneous, $\partial(\tau f)=\partial f . \tau$ is an automorphism of the category $A$-MOD, or, equally well, of $A$-Mod. $\tau A$ is an $A$-module, but not a ring. $\tau M \approx M$ in $A$-Mod $\Rightarrow M_{1} \approx M_{0}$ as $A_{0}$-modules, whereas, at least if $A=\left(A_{0}\right)$, we have $\tau M \approx M$ in $A$-MOD for all $M$.

If $A$ is a graded ring, $A^{\circ}$ denotes the usual opposite ring, and $A^{\prime}$ denotes the additive group of $A$ with multiplication redefined by $a \cdot b=-1^{a b} a b$ for $a, b \in h A$, and by distributivity in general. $A^{\prime}$ is a ring: $a \cdot(b \cdot c)=-1^{a b+a c+b c} a b c=(a \cdot b) \cdot c$. If $M$ is a graded $A$-module we define an action of $A^{\prime}$ on the additive group of $M$ by $a \cdot x=-1^{a x} a x$ for $a \in h A, x \in h M$, and by "distributivity" in general. The result is an $A^{\prime}$-module $\left(a \cdot(b \cdot x)=-1^{a b+a x+b x} a b x=(a \cdot b) \cdot x\right)$, which we christen $M^{\prime}$. 
If $f \in \operatorname{HOM}_{A}(M, N)$ and we view $f$ as a map $M^{\prime} \rightarrow N^{\prime}$, we have $f(a \cdot x)$ $=-1^{a x+a f} a(f x)=-1^{a x+a f+a(f x)} a \cdot(f x)$. This latter is just $a \cdot(f x)$, for $\partial(f x)=\partial f+\partial x$. Thus,

(1.4) Proposition. The functor $A$-Mod ' $\rightarrow A^{\prime}$-Mod is a category isomorphism. The "identity" is a natural isomorphism $\operatorname{HOM}_{A}(M, N) \approx \operatorname{Hom}_{\left|A^{\prime}\right|}\left(\left|M^{\prime}\right|,\left|N^{\prime}\right|\right)$, so that $A$-MOD " is" the category whose objects are graded $A^{\prime}$-modules, with morphisms $\operatorname{Hom}_{\left|A^{\prime}\right|}(||$,$) .$

Proof. The first statement is clear. Since the computation above shows $f(a \cdot x)$ $=a \cdot(f x)$ regardless of $\partial f$, we do not get an isomorphism $A$-MOD $\rightarrow A^{\prime}$-MOD, but only an inclusion (easily seen to be an equality) $\operatorname{HOM}_{A}(M, N) \subset \operatorname{Hom}_{\left|A^{\prime}\right|}\left(\left|M^{\prime}\right|,\left|N^{\prime}\right|\right)$.

REMARK. The word graded in the second part of (1.4) is important. If the given isomorphism came from an equivalence $A$-MOD $\rightarrow\left|A^{\prime}\right|$-Mod (=the ordinary category of ungraded modules) we would be contradicting the observation that $A$-MOD is, in general, not an abelian category. The forgetful functor $|'|$ is far from surjective on objects.

(1.5) Proposition. $\operatorname{Hom}_{A}(M, \tau N)=\operatorname{Hom}_{A}(\tau M, N)=\operatorname{HOM}_{A^{\prime}}\left(M^{\prime}, N^{\prime}\right)_{1}$.

(1.6) Corollary. (a) $\operatorname{Hom}_{A}(M, \tau N) \approx \operatorname{HOM}_{A}(M, N)_{1}$.

(b) If $A=\left(A_{0}\right), \mathrm{HOM}_{A}(M, N) \approx \mathrm{Hom}_{|A|}(|M|,|N|)$ as graded abelian groups.

If $A$ is a graded ring we let $A^{*}$ denote $A^{\circ \prime}$ (or, what is the same, $A^{\circ}$ ). For any subset $S$ of $h A$ we let $C_{A}(S)$ denote $\left\langle\left\{a \in h A \mid a b=-1^{a b} b a \forall b \in S\right\}\right\rangle$; for any $S$, this is a (graded) subring. We let CENTER $A$ denote $C_{A}(A)$, and we call $A$ commutative if CENTER $A=A$, i.e., if $A$ and $A^{*}$ coincide. Center $A$ will denote the degree zero term of CENTER $A\left({ }^{2}\right)$. Note that CENTER $A$, Center $A$, Center $|A|$, and Center $A_{0}$ are, in general, distinct, although Center $A$ is contained in each.

If $K$ is a commutative graded ring, a $K$-algebra is a homomorphism $K \rightarrow A$ whose image lies in CENTER $A$. (If $K=\left(K_{0}\right)$, " $A$ is a $K$-algebra" implies " $|A|$ is a $|K|$-algebra", but if $K_{1} \neq 0,|K|$ need not be commutative.) A homomorphism of $K$-algebras is a homomorphism $A \rightarrow B$ of graded rings such that

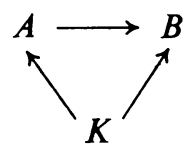

commutes.

If $A$ is a $K$-algebra and $M$ and $N$ are $A$-modules, $(t f) x=t(f x)$ makes $\operatorname{HOM}_{A}(M, N)$ a $K$-module.

(2) The general notational convention, as is now apparent, is that an object spelled with capital letters is graded, and the same object with only the initial letter capitalized refers to its degree zero term. However, we prefer the term "graded ring" to the more logical alternative, "RING".... Another bit of notational philosophy, also clear by now, is that the price of moving $x$ past $y$ is the sign $-1^{x y}$, as usual. 
(1.7) Proposition. If $A$ is a $K$-algebra (e.g. $K=$ CENTER $A$, or $(Z)$, for any graded ring $A), f \mapsto f(1)$ defines an isomorphism $\operatorname{HOM}_{A}(A,) \rightarrow$ "identity" of functors from $A$-MOD to $K$-MOD. Evaluating at $A$ itself we have an isomorphism $\operatorname{END}_{A}(A) \rightarrow A^{*}$ of $K$-algebras.

Proof. For any $A$-module $N, x \mapsto f_{x}$, given on homogeneous elements by $f_{x}(a)=-1^{a x} a x$, defines an inverse, $N \rightarrow \operatorname{HOM}_{A}(A, N)$. The rest is routine.

Let $A$ and $B$ be $K$-algebras, $K$ commutative. Although $|A|$ and $|B|$ may fail to be $|K|$-algebras, we can view them as (right or left) $|K|$-modules, so the abelian group $|A| \otimes_{|K|}|B|$ is defined. Grade $|A| \otimes_{|K|}|B|$ by letting $\left[|A| \otimes_{|K|}|B|\right]_{i}$ be the subgroup generated by $\{a \otimes b \mid a \in h A, b \in h B, \partial a+\partial b=i(\bmod 2)\}$, and define a product by the rule $(a \otimes b)\left(a^{\prime} \otimes b^{\prime}\right)=-1^{b a^{\prime}}\left(a a^{\prime} \otimes b b^{\prime}\right)$ for homogeneous generators. $|A| \otimes_{|K|}|B|$, with the grading and multiplication just described, we christen $A \otimes_{K} B$.

$A \otimes_{K} B$ is a graded ring, and $x \mapsto x \otimes 1=1 \otimes x$ makes $A \otimes_{K} B$ a $K$-algebra. The latter follows from

$$
\{a \otimes b \mid a \in h \text { CENTER } A, b \in h \text { CENTER } B\} \subset h \text { CENTER } A \otimes_{K} B,
$$

which is immediate. $x \mapsto x \otimes 1$ is an algebra homomorphism because $\partial 1=0$ implies that $(x \otimes 1)(y \otimes 1)=x y \otimes 1$ and $\partial(x \otimes 1)=\partial x$. The same remark shows that the subalgebras $A \otimes 1$ and $1 \otimes B$ commute in $A \otimes B:(x \otimes 1)(1 \otimes y)$ $=x \otimes y=-1^{\partial(x \otimes 1) \partial(1 \otimes y)}(1 \otimes y)(x \otimes 1) . A \otimes_{K} B$ is universal for this property: if $f: A \rightarrow C$ and $g: B \rightarrow C$ are $K$-algebra homomorphisms whose images commute in $C$, there is a unique $K$-algebra homomorphism $A \otimes_{K} B \rightarrow C$ which makes

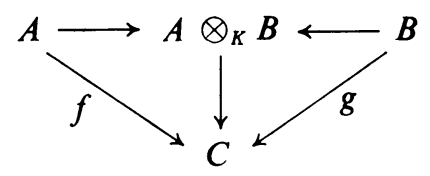

commute.

(1.8) LeMmA. If $A$ and $B$ are $K$-algebras there are isomorphisms

(i) $A \otimes B \rightarrow B \otimes A$,

(ii) $(A \otimes B)^{\prime} \rightarrow A^{\prime} \otimes B^{\prime}$,

(iii) $(A \otimes B)^{\circ} \rightarrow A^{\circ} \otimes B^{\circ}$,

(iv) $(A \otimes B)^{*} \rightarrow A^{*} \otimes B^{*}$,

(v) $A \otimes K \rightarrow A$,

of $K$-algebras, where $\otimes$ means $\otimes_{K}$.

Proof. Define the maps by letting the image of a homogeneous generator $a \otimes b$ be $-1^{a b} b \otimes a$ in (i), $-1^{a b} a \otimes b$ in (ii) and (iii), $a \otimes b$ itself in (iv), and $a b$ in (v). It suffices to check that these define homomorphisms and this is a routine calculation in each case.

If $A$ and $B$ are $K$-algebras and $M$ (resp. $N$ ) is an $A$ (resp. $B$ ) module, we define $M \otimes_{K} N$ in the obvious way: grade $|M| \otimes_{|K|}|N|$ as before, and make the result 
an $A \otimes_{K} B$-module by defining $(a \otimes b)(x \otimes y)=-1^{b x}(a x \otimes b y)$ for homogeneous generators.

(1.9) Lemma. Let $A$ and $B$ be K-algebras, let $M$ (resp. $N$ ) be an $A$-(resp. B-) module, and write $\otimes$ for $\otimes_{K}$. There are isomorphisms

(i) $\tau M \otimes N \approx \tau(M \otimes N) \approx M \otimes \tau N$ and $\tau M \otimes \tau N \approx M \otimes N$ of $A \otimes B$-modules,

(ii) $(M \otimes N)^{\prime} \approx M^{\prime} \otimes N^{\prime}$ of $(A \otimes B)^{\prime}=A^{\prime} \otimes B^{\prime}$-modules, and

(iii) $M \otimes N \approx N \otimes M$ of $A \otimes B=B \otimes A$-modules.

Note that when $K=\left(K_{0}\right), M_{i}$ and $N_{i}$ are $|K|$-modules, $i=0,1$, so that $M \otimes_{K} N=\coprod_{i, j=0,1} M_{i} \otimes_{K} N_{j}$ as graded $K$-modules. Explicitly, $[M \otimes N]_{0}$ $=\left(M_{0} \otimes N_{0}\right) \oplus\left(M_{1} \otimes N_{1}\right)$ and $[M \otimes N]_{1}=\left(M_{0} \otimes N_{1}\right) \oplus\left(M_{1} \otimes N_{0}\right)$.

By way of example we introduce here several algebras we will need later. Let $k$ be commutative and concentrated in degree zero, and let $x, y \in k$.

$k\langle x\rangle$ will denote $|k| 1 \oplus|k| t$ with $t^{2}=x$, i.e., $k[X] /\left(X^{2}-x\right)$ with the indicated grading. (Note that $k\langle x\rangle^{*}=k\langle-x\rangle$.) $k\{x\}$ will denote $k[X] /\left(X^{2}+X-x\right)$, concentrated in degree zero.

$$
\left(\frac{x, y}{k}\right)
$$

will denote the "graded quaternion algebra" $k\langle x\rangle \otimes_{k} k\langle y\rangle$; thus

$$
\left(\frac{x, y}{k}\right)
$$

has a $k$-basis $\{1, s, t, s t\}$ with $s$ and $t$ of degree one, and relations $s^{2}=x, t^{2}=y$, $s t+t s=0$.

$$
\left[\frac{x, y}{k}\right]
$$

will denote the algebra with the same basis, and defining relations $s^{2}=x, t^{2}=y$ and $s t+t s=1$. (This latter is the appropriate notion of "graded quaternion algebra" when $2=0$ in $k$; if we forget its grading,

$$
\left[\frac{x, y}{k}\right]
$$

is isomorphic to the algebra ( $x, x y$ ] considered in [9] (cf. [9, pp. 188 and 198-199]), which is central simple if $k$ is a field of characteristic two.)

Let $A$ be a $k$-algebra, $k=\left(k_{0}\right)$ commutative, and let $T$ denote $k\langle 1\rangle$. If $N$ is an $|A|$-module, $T \otimes(N)=(1 \otimes N) \oplus(t \otimes N)$ is a $T \otimes(|A|)$-module. In particular $T \otimes(|A|)=(1 \otimes|A|) \oplus(t \otimes|A|)$, and $a \mapsto t^{\partial a} \otimes a$ (for $a \in h A$ ) defines a $k$ algebra homomorphism $f: A \rightarrow T \otimes(|A|)$, and $T \otimes(N)$ is an $A$-module via $f$.

(1.10) Proposition. With notation as above, the functors

$$
A-\operatorname{Mod} \underset{T \otimes(~)}{\stackrel{||}{\leftrightarrows}}|A|-\operatorname{Mod}
$$


are adjoint: for any $|A|$-module $N$ and $A$-module $M$,

$$
\operatorname{Hom}_{|A|}(|M|, N) \approx \operatorname{Hom}_{A}(M, T \otimes(N)) .
$$

Proof. If $g \in \operatorname{Hom}_{|A|}(|M|, N)$, define $\tilde{g}: M \rightarrow T \otimes(N)$ by $x \mapsto t^{\partial x} \otimes g x$. Conversely, if $h \in \operatorname{Hom}_{A}(M, T \otimes(N)), t \mapsto 1$ induces a map from $|M|$ to $N$. These are easily seen to be inverse isomorphisms.

(1.11) Proposition and Definition. A graded $A$-module $M$ is projective if it satisfies the following conditions, which are equivalent:

(i) $\operatorname{Hom}_{A}(M$, ) is exact on $A$-Mod.

(ii) $\mathrm{HOM}_{A}(M$,$) is exact on A$-Mod.

(iii) $\mathrm{HOM}_{A}(M$,$) is exact on A$-MOD.

(iv) $\operatorname{Hom}_{|A|}(|M|$, ) is exact on $|A|$-Mod.

Proof of equivalence. (iii) $\Rightarrow$ (ii) $\Rightarrow$ (i) are clear, and (i) $\Rightarrow$ (iv) follows from (1.10): the existence of an adjoint implies that $T \otimes(~)$ is exact, and consequently that | preserves projectives. To complete the proof we show (iv) $\Rightarrow$ (i) $\Rightarrow$ (iii).

(iv) $\Rightarrow$ (i). Given a diagram

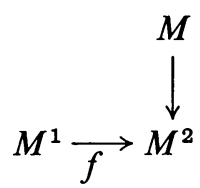

in $A$-Mod with $f$ surjective, there is by (iv) an arrow $g: M \rightarrow M^{1}$ making the triangle commute. Unfortunately, $g$ need not respect the grading. However, if we define $h(x)$, for $x \in M_{i}$, to be the projection of $g(x)$ in $M_{i}^{1}$, we get an arrow $h: M \rightarrow M^{1}$ in $A$-Mod without spoiling commutativity of the triangle.

(i) $\Rightarrow$ (iii). (i) implies that $\operatorname{Hom}_{A^{\prime}}\left(M^{\prime}\right.$, ) is exact on $A^{\prime}$-Mod, and therefore that $\operatorname{Hom}_{\left|A^{\prime}\right|}\left(\left|M^{\prime}\right|\right.$, ) is exact on $\left|A^{\prime}\right|-\operatorname{Mod}$ (by (i) $\Rightarrow$ (iv), which we have already proved). With (1.4), this yields (iii).

By a free module over a graded ring $A$ we mean one with a homogeneous basis, i.e., of the form $F=A^{(I)} \oplus[\tau A]^{(J)}$, i.e., $F_{0}=A_{0}^{(I)} \oplus A_{1}^{(J)}$ and $F_{1}=A_{0}^{(J)} \oplus A_{1}^{(I)}$. As usual, $M$ is finitely presented if there is an exact sequence $F^{2} \rightarrow F^{1} \rightarrow M \rightarrow 0$ with $F^{1}$ and $F^{2}$ free of finite rank.

(1.12) Corollary. (i) An A-module is projective if and only if it is a direct summand of a free A-module.

(ii) A finitely generated projective A-module is finitely presented.

2. Bimodules and separable algebras. By a right $A$-module $M$ we mean a right $|A|$-module which is additively a direct sum $M=M_{0} \oplus M_{1}$ satisfying $M_{\mathfrak{i}} A_{j} \subset M_{\mathfrak{i}+j}$ $(i, j \in Z / 2 Z)$. For right $A$-modules $M$ and $N$ we let $\mathbf{H O M}_{A}(M, N)_{j}$ denote the subgroup $\left\{f \mid f\left(M_{i}\right) \subset N_{i+j}\right\}$ of $\operatorname{Hom}_{|A|}(|M|,|N|)$, and we write $\operatorname{Hom}_{A}(M, N)$ for $\mathbf{H O M}_{A}(M, N)_{0}$ and $\mathbf{H O M}_{A}(M, N)$ for the graded abelian group $\operatorname{Hom}_{A}(M, N) \oplus \operatorname{HOM}_{A}(M, N)_{1}$. MOD- $A$ and Mod- $A$ will mean the obvious things. 
(2.1) LeMMA. $\operatorname{HOM}_{A}(M, N) \approx \operatorname{Hom}_{A}(M \oplus \tau M, N) \approx \operatorname{Hom}_{|A|}(|M|,|N|)$.

(Again, the isomorphism does not come from an equivalence MOD- $A \rightarrow \operatorname{Mod}-|A|$.)

If $M$ is a right $A$-module, the definition $a x=-1^{a x} x a$ makes $M$ a left $A^{*}$-module, and conversely. This procedure matches $\mathbf{H O M}_{A}$ and $\mathbf{H O M}_{A^{*}}-f(x a)$ $=-1^{a x+a f} a(f x)=-1^{a x+a f+a(f x)}(f x) a=(f x) a$-so that MOD- $A$ and $A^{*}$-MOD are isomorphic categories.

If $M$ is simultaneously a left $A$-module and a right $B$-module, where $A$ and $B$ are $K$-algebras, we call $M$ an $A_{K} B$-bimodule provided

$$
\begin{array}{ll}
\text { (a) }(a x) b=a(x b) & (a \in A, x \in M, b \in B), \\
\text { (b) } c x=-1^{c x} x c & (x \in h M, c \in h K) .
\end{array}
$$

If $M$ is an $A_{K} B$-bimodule, $(a \otimes b) x=-1^{b x} a x b$ makes $M$ a left $A \otimes_{K} B^{*}$-module, and conversely if $M$ is a left $A \otimes B^{*}$-module, $a x=(a \otimes 1) x$ and $x b=-1^{b x}(1 \otimes b) x$ make $M$ an $A_{K} B$-bimodule. These recipes are inverses, so that the two notions coincide.

We will sometimes write "the bimodule ${ }_{A} M_{B}$ " instead of "the $A_{K} B$-bimodule $M$ " when the ground ring $K$ is fixed by the context. Our real interest is in $A_{K} A$ bimodules, i.e., modules over the enveloping algebra $A_{K}^{e}=A \otimes_{K} A^{*}$ of $A$. $A$ is itself an $A_{K}^{e}$-module, and $a \otimes b \mapsto a b$ defines an epimorphism $\varphi: A_{K}^{e} \rightarrow A$, and thereby an exact sequence

$$
E_{K}(A): 0 \longrightarrow J_{K}(A) \longrightarrow A_{K}^{e} \stackrel{\varphi}{\longrightarrow} A \longrightarrow 0
$$

in $A_{K}^{e}$-Mod. We will usually omit the subscripts referring to the ground ring, and when $A$ is fixed we will write simply $J$ for $J_{K}(A)$.

Define $\delta: A \rightarrow J$ by $a \mapsto a \otimes 1-1 \otimes a$. $\delta$ is $K$-linear but not $A^{e}$-linear.

(2.3) Lemma. The image of $\delta$ generates $J$ as a left ideal in $A^{e}$, and $\delta$ satisfies $\delta(a b)=(\delta a) b+a(\delta b)(a, b \in A)$.

The proof is a straightforward imitation of the ungraded analog $[3$, Lemma III.1.2].

Let $M$ be an $A^{e}$-module. Define

$\operatorname{DER}_{K}(A, M)=\left\langle\left\{d \in h \operatorname{HOM}_{K}(A, M) \mid d(a b)=(d a) b+(-1)^{a d} a(d b) \forall a, b \in h A\right\}\right\rangle$.

This is a $K$-submodule of $\operatorname{HOM}_{K}(A, M)$. Its degree zero term is denoted $\operatorname{Der}_{K}(A, M)$, and elements of DER are called derivations.

(2.4) Lemma. If $d \in \operatorname{DER}_{K}(A, M)$ and $f \in \operatorname{HOM}_{A^{e}}(M, N)$ then $f d \in \operatorname{DER}_{K}(A, N)$.

It suffices to prove this when $d$ and $f$ are homogeneous. This is a simple computation (note that $\partial(f d)=\partial f+\partial d)$.

Lemma (2.3) shows that $\delta \in \operatorname{Der}_{K}(A, J)$. For $x \in M$, define

$$
d_{x}=f_{x} \delta \in \operatorname{DER}_{K}(A, M),
$$


where $f_{x}$ is the element of $\operatorname{HOM}_{A^{e}}\left(A^{e}, M\right)$ associated by (1.7) to $x \in M$. For homogeneous $x$ and $a, d_{x} a=f_{x}(a \otimes 1-1 \otimes a)=-1^{a x}(\delta a) x=-1^{a x} a x-x a$. Derivations of the form $d_{x}$ for some $x \in M$ are called inner derivations; they form a $K$-submodule $\operatorname{INDER}_{K}(A, M)$ of $\operatorname{DER}_{K}(A, M)$.

(2.5) Proposition. $f \mapsto f \delta$ induces an isomorphism $\operatorname{HOM}_{A^{e}}(J,) \rightarrow \operatorname{DER}_{K}(A$, ) of functors $A^{e}$-MOD $\rightarrow K$-MOD.

Proof. It suffices to show that $\operatorname{HOM}_{A^{e}}(J, M) \rightarrow \operatorname{DER}_{K}(A, M)$ is an isomorphism for every $M$. Injectivity follows from the fact that the image of $\delta$ generates $J$, (2.3). For surjectivity, suppose $d \in h \operatorname{DER}_{K}(A, M)$. Then $a \otimes b \mapsto-(-1)^{a d} a(d b)$ defines a $K$-linear map $f: A^{e} \rightarrow M$ which is homogeneous of degree $\partial d$. Since $f(\delta a)$ $=d a$, it suffices to show that the restriction to $J$ of $f$ is actually $A^{e}$-linear. This is an unpleasant, but straightforward, computation.

We need one further definition for our $A^{e}$-module $M$. We let $M^{A}$ denote the $K$-submodule $\{x \in M \mid(\delta a) x=0 \forall a \in A\}$ of $M$. Since $(\delta a) x=(a \otimes 1) x-(1 \otimes a) x$, the definition, if we view $M$ as an $A$ - $A$-bimodule, is

$$
M^{A}=\left\langle\left\{x \in h M \mid a x=-1^{a x} x a \forall a \in h A\right\}\right\rangle .
$$

Now a glance at $E(A)$ shows that $\varphi$ identifies $\operatorname{HOM}_{A^{e}}(A, M)$ with

$$
\left\{f \in \operatorname{HOM}_{A^{e}}\left(A^{e}, M\right) \mid J \subset \operatorname{ker} f\right\},
$$

and (1.7) allows us to identify this with $\left\{x \in M \mid J \subset \operatorname{ker} f_{x}\right\}$, the "right annihilator" of $J$ in $M$. Since the image of $\delta$ generates $J$, the initial definition of $M^{A}$ shows that it is this right annihilator. We have proved:

(2.6) Proposition. The diagram

$$
\begin{gathered}
M \approx \operatorname{HOM}_{A^{e}}\left(A^{e}, M\right) \\
\cup \cup \cup \cup \\
M^{A} \approx \operatorname{HOM}_{A^{e}}(A, M)
\end{gathered}
$$

commutes, where the right-hand inclusion is induced by $\varphi$.

We now have, for any $A^{e}$-module $M$, a commutative diagram

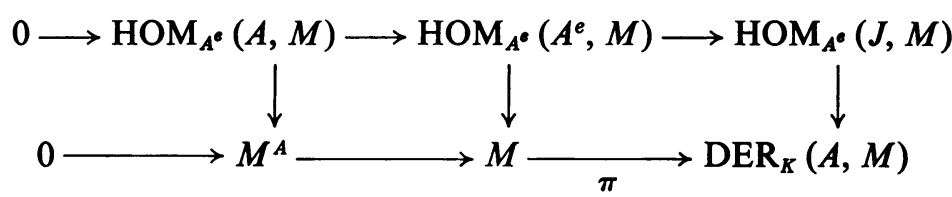

in which the verticals are isomorphisms. The top row is exact (it is just $\operatorname{HOM}_{A^{e}}(E(A), M)$ ), and $\pi$ is the map which assigns to $x \in M$ the inner derivation $d_{x}$. Let $\operatorname{EXT}_{A^{e}}^{n}(A, M)$ denote "as usual" the right derived functors of $\operatorname{HOM}_{A^{e}}(A, M) \approx M^{A}$. Then 
(2.8) Proposition. If $M$ is an $A^{e}$-module there is an exact sequence $0 \rightarrow M^{A}$ $\rightarrow M \rightarrow \operatorname{DER}_{K}(A, M) \rightarrow \operatorname{EXT}_{A}^{1} e(A, M) \rightarrow 0$ and it induces

$$
\operatorname{EXT}_{A}^{1} e(A, M) \approx \operatorname{DER}_{K}(A, M) / \operatorname{INDER}_{K}(A, M)
$$

In particular this applies to $M=A$ and $M=A^{e} . A^{A}$ is just CENTER $A$, and $A^{e^{A}}$ is the right annihilator of $J(A)$ in $A^{e}$. Note that $\varphi\left(A^{e^{A}}\right) \subset A^{A}$, for $M \mapsto M^{A}$ is the functor $\operatorname{HOM}_{A^{e}}(A$,$) , and \varphi \in \operatorname{Hom}_{A^{e}}\left(A^{e}, A\right)$ maps $\operatorname{HOM}_{A^{e}}\left(A, A^{e}\right)$ to $\operatorname{HOM}_{A^{e}}(A, A)$. In fact,

(2.9) LemMA. $\varphi\left(A^{e^{A}}\right)$ is an ideal in $A^{A}$.

We omit the simple proof. It is worth noting, however, that $\varphi\left(A^{e A}\right)$ is not a left ideal in $A$ itself.

(2.10) Theorem And Definition. A K-algebra $A$ is separable if it satisfies the following conditions, which are equivalent:

(1) $E_{K}(A)$ is a split exact sequence of $A_{K}^{e}$-modules.

(2) $A$ is a projective $A_{K}^{e}$-module.

(2') $|A|$ is a projective $\left|A_{K}^{e}\right|$-module.

(3) $\varphi\left(A^{e^{A}}\right)=A^{A}$ (cf. 2.9).

(4) $\delta: A \rightarrow J_{K}(A)$ is an inner derivation.

(5) Every $K$-derivation on $A$ (to an $A_{K}^{e}$-module) is inner.

(6) $M \mapsto M^{A}$ is an exact functor of $A_{K}^{e}$-modules.

(7) For any $A_{K}^{e}$-module $M$, the sequence

$$
\operatorname{HOM}_{A^{e}}\left(E_{K}(A), M\right) \approx\left(0 \rightarrow M^{A} \rightarrow M \rightarrow \operatorname{DER}_{K}(A, M) \rightarrow 0\right)
$$

is exact and splits as CENTER $A^{e}$-modules.

(8) Same as (7) but for $M=J_{K}(A)$ only.

Proof of equivalence. Most of the implications follow from (2.7) and (2.8). (1) $\Leftrightarrow$ (3) follows from the commutative diagram

$$
\begin{aligned}
& A^{e^{A}} \stackrel{\varphi}{\longrightarrow} A^{A} \\
& \sim \text { ح } \\
& \operatorname{HOM}_{A^{e}}\left(A, A^{e}\right) \underset{\mathrm{HOM}_{A^{e}}(A, \varphi)}{\longrightarrow} \operatorname{HOM}_{A^{e}}(A, A)
\end{aligned}
$$

The equivalence of (4) and (5) is analogous to the equivalence of (7) and (8); since clearly $(5) \Leftrightarrow(1) \Leftrightarrow(7)$, it is simplest to prove (4) $\Leftrightarrow(1)$ and $(8) \Leftrightarrow(1)$. Assuming (8), for example, we have that $\mathrm{HOM}_{A^{e}}\left(A^{e}, J\right) \rightarrow \operatorname{HOM}_{A^{e}}(J, J)$ is surjective; hence the identity map on $J$ extends to $A^{e}$, so that $E(A)$ splits. Similarly, for (4) $\Leftrightarrow(1)$ we can consider the commutative square

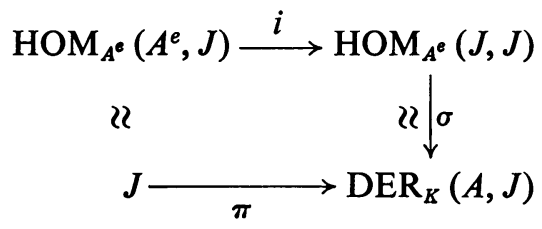


(from (2.7)); clearly $\sigma$ induces $\operatorname{Im} i \approx \operatorname{Im} \pi=\operatorname{INDER}_{K}(A, J)$. Now $\delta$ is the image, under $\sigma$, of the identity map on $J$, and therefore $\delta$ is inner if and only if the identity on $J$ extends to $A^{e}$, hence if and only if $E(A)$ splits.

(2.11) Corollary. If $p: A \rightarrow B$ is an epimorphism of $K$-algebras and $A$ is $K$ separable then $B$ is $K$-separable and CENTER $B=p($ CENTER $A)$.

In particular, any quotient of $K$ is $K$-separable.

REmark. The equivalence of (2) and (2') in (2.10) does not mean that $A$ is $K$-separable if and only if $|A|$ is $|K|$-separable. In the first place, as we have seen, $|A|$ may not even be a $|K|$-algebra-indeed $|K|$ need not be commutative. But even if $|A|$ is a $|K|$-algebra, $|K|$-separability of $|A|$ would mean projectivity of $|A|$ as an $|A|^{e}$-module, and $|A|^{e} \neq\left|A^{e}\right|$ in general.

Added in proof. M. Orzech points out that when $K=\left(K_{0}\right), A$ is $K$-separable $\Leftrightarrow|A|$ is $|K|$-separable. For existence of $f \in \operatorname{Hom}_{A^{e}}\left(A, A^{e}\right)$ splitting $\varphi: A^{e} \rightarrow A$ is equivalent to existence of $x=\sum a_{i} \otimes b_{i} \in A^{e}$ with

(i) $\sum a_{i} b_{i}=1(f$ splits $\varphi)$,

(ii) $\sum(a \otimes 1-1 \otimes a)\left(a_{i} \otimes b_{i}\right)=0$ for all $a \in A\left(x \in A^{e^{A}}\right)$, and

(iii) $\partial\left(a_{i} \otimes b_{i}\right)=0$ for each $i\left(x \in A_{0}^{e}\right)$.

Now (ii) is unchanged if we read it in $|A|^{e}=|A| \otimes_{|K|}|A|^{\circ}$, since the sign $-1^{a\left(a_{i}+b_{i}\right)}$ evaporates because of (iii), and with (i) (read in $|A|$ ) it is equivalent to $|K|$ separability of $|A|$. Conversely, an element $\sum a_{i} \otimes b_{i} \in|A|^{e}$ satisfying (i) and (ii) in the ungraded sense can be read as an element of $A^{e}$ satisfying (i) and (ii), and if it is not homogeneous of degree zero we can replace it by its projection in $A_{0}^{e}$.

3. Lemmas on separability; graded Morita theory. In this section we assemble a host of lemmas, translations into our graded context of results well-known in the ungraded situation. Since the proofs as well as the statements are straightforward imitations of their ungraded counterparts, we omit them. The ungraded theories are treated in Chapter III of [3] and $\$ \$ 1-3$ of [1] (separability lemmas), and Chapter II of [3] and Chapter II of [2] (Morita theory).

(3.1) Lemma. Let $A_{i}$ be $K$-algebras and let $M_{i}$ (resp. $N_{i}$ ) be a right (resp. left) $A_{i}$ module, $i=1,2$. Let $A$ denote $A_{1} \otimes_{K} A_{2}$ and let $K_{i}=$ CENTER $A_{i}$. Then

$$
\left(m_{1} \otimes m_{2}\right) \otimes\left(n_{1} \otimes n_{2}\right) \mapsto-1^{n_{1} m_{2}}\left(m_{1} \otimes n_{1}\right) \otimes\left(m_{2} \otimes n_{2}\right)
$$

defines an isomorphism

$$
f:\left(M_{1} \otimes_{K} M_{2}\right) \otimes_{A}\left(N_{1} \otimes_{K} N_{2}\right) \rightarrow\left(M_{1} \otimes_{A_{1}} N_{1}\right) \otimes_{K}\left(M_{2} \otimes_{A_{2}} N_{2}\right)
$$

of $C=K_{1} \otimes_{K} K_{2}$-modules. If $M_{i}$ and $N_{i}$ are $K_{i}$-algebras, $f$ is an isomorphism of $C$ algebras. In either case the isomorphism is natural in all variables for module morphisms of degree zero.

(3.2) Corollary. Let $K_{i}$ be commutative $K$-algebras and let $A_{i}$ be a $K_{i}$-algebra, $i=1,2$. There is a natural isomorphism

$$
\left(A_{1} \otimes_{K} A_{2}\right)_{C}^{e} \rightarrow A_{1_{K_{1}}}^{e} \otimes_{K} A_{2_{K_{2}}}^{e} \text { of } C=K_{1} \otimes_{K} K_{2} \text {-algebras. }
$$


(3.3) CoRollary. If $A$ and $L$ are $K$-algebras, $L$ commutative, there is a natural isomorphism $\left(L \otimes_{K} A\right)_{L}^{e} \rightarrow L \otimes_{K} A_{K}^{e}$.

Let $K_{i}$ be commutative $K$-algebras, let $A_{i}$ be a $K_{i}$-algebra, and let $M_{i}$ and $N_{i}$ be $A_{i}$-modules, $i=1$, 2. Define a map $\psi$ from $\operatorname{HOM}_{A_{1}}\left(M_{1}, N_{1}\right) \otimes_{K} \operatorname{HOM}_{A_{2}}\left(M_{2}, N_{2}\right)$ to $\mathrm{HOM}_{A_{1} \otimes_{K} A_{2}}\left(M_{1} \otimes_{K} M_{2}, N_{1} \otimes_{K} N_{2}\right)$ as follows. For $f_{i} \in h \operatorname{HOM}_{A_{i}}\left(M_{i}, N_{i}\right)$ and $x_{i} \in h M_{i}$, set $\left(\psi\left(f_{1} \otimes f_{2}\right)\right)\left(x_{1} \otimes x_{2}\right)=-1^{f_{2} x_{1}} f_{1} x_{1} \otimes f_{2} x_{2}$. One sees easily that $\psi$ is well defined and $K_{1} \otimes_{K} K_{2}$-linear, and that its image is indeed in

$$
\mathrm{HOM}_{A_{1} \otimes_{K} A_{2}}\left(M_{1} \otimes_{K} M_{2}, N_{1} \otimes_{K} N_{2}\right) \text {. }
$$

(3.4) LEMMA. $\psi$ is a $K_{1} \otimes_{K} K_{2}$-isomorphism in either of the following situations:

(i) $M_{i}$ is a finitely generated projective $A_{i}$-module, $i=1,2$.

(ii) $M_{1}$ is a finitely generated projective $A_{1}$-module, $M_{2}$ is a finitely presented $A_{2}$-module, $A_{1}$ is $K$-flat, and $N_{1}$ is either $K$-flat or finitely generated and projective over $A_{1}$.

In either case, the isomorphism is natural in all variables for module morphisms of degree zero. (A $K$-module $M$ is said to be $f l a t$ if $M \otimes_{K}$ is an exact functor on $K$-Mod.)

(3.5) Corollary. Let $P_{i}$ be finitely generated projective $A_{i}$-modules, where $A_{i}$ are $K_{i}$-algebras and $K_{i}$ are commutative $K$-algebras, $i=1,2$. Then there is a natural isomorphism $\mathrm{END}_{A_{1}}\left(P_{1}\right) \otimes_{K} \operatorname{END}_{A_{2}}\left(P_{2}\right) \rightarrow \operatorname{END}_{A_{1} \otimes_{K} A_{2}}\left(P_{1} \otimes_{K} P_{2}\right)$ of $K_{1} \otimes_{K} K_{2}-$ algebras.

(3.6) LeMMA. If a $K$-algebra $A$ is finitely presented as a $K$-module, it is finitely presented as an $A_{K}^{e}$-module.

The proof of (3.6) is a direct translation of the proofs of (2.1) and (2.2) of Chapter III of [3].

(3.7) Corollary. Let $A_{i}$ be $K_{i}$-algebras, $K_{i}$ commutative $K$-algebras, and let $M_{i}$ be an $A_{i_{\mathrm{K}}}^{e}$-module, $i=1,2$. There is a natural isomorphism

$$
M_{1}^{A_{1}} \otimes_{K} M_{2}^{A_{2}} \rightarrow\left(M_{1} \otimes_{K} M_{2}\right)^{A_{1} \otimes_{K} A_{2}}
$$

(as $K_{1} \otimes_{K} K_{2}$-modules) in either of the following situations:

(i) $A_{i}$ is $K_{i}$-separable, $i=1,2$.

(ii) $A_{1}$ is $K_{1}$-separable and $K$-flat, $A_{2}$ is finitely presented (as $A_{2_{K_{2}}}^{e}$ or as $K_{2}$ module), and $M_{1}$ is either $K$-flat or finitely generated and projective over $A_{1_{\Sigma_{1}}}^{e}$.

(3.8) Corollary. (i) If $A_{i}$ is $K_{i}$-separable, $i=1,2$, then $A_{1} \otimes_{K} A_{2}$ is $K_{1} \otimes_{K} K_{2}$ separable, and its CENTER is $A_{1}^{A_{1}} \otimes_{K} A_{2}^{A_{2}}$.

(ii) If $A$ is a separable $K$-algebra and $L$ is a commutative $K$-algebra then $L \otimes_{K} A$ is a separable L-algebra and its CENTER is $L \otimes_{K} A^{A}$.

(3.9) Corollary. Let $L$ and $A$ be $K$-algebras with $L K$-flat. Then

(i) If $M$ and $N$ are A-modules and $M$ is finitely presented, there is a natural isomorphism

$$
L \otimes_{K} \operatorname{HOM}_{A}(M, N) \rightarrow \operatorname{HOM}_{L} \otimes_{K} A\left(L \otimes_{K} M, L \otimes_{K} N\right) .
$$


(ii) If $L$ is commutative and $A$ is finitely presented as $K$-or $A_{K}^{e}$-module then for any $A_{K}^{e}$-module $N$ there is a natural isomorphism

$$
L \otimes_{K} N^{A} \rightarrow\left(L \otimes_{K} N\right)^{L} \otimes_{K}^{A} .
$$

(3.10) Corollary. Let $A$ and $L$ be $K$-algebras, $L$ commutative. Assume that $L$ is faithfully $K$-flat, that is, a sequence $E$ in $K$-Mod is exact if and only if $L \otimes_{K} E$ is exact. Suppose also that $A$ is finitely presented as $K$ - or $A_{K}^{e}$-module. Then $L \otimes_{K} A$ is $L$-separable $\Rightarrow A$ is $K$-separable.

We turn next to the so-called Morita theory. Fix a commutative ground ring $k=\left(k_{0}\right)$ and let $A$ be a (graded) $k$-algebra.

(3.11) Lemma And Definition. Let $M$ be $a$ (graded) A-module. $M$ is a GENERATOR (in A-MOD) if it satisfies the following conditions, which are equivalent:

(1) $\mathrm{HOM}_{A}(M$,$) is faithful on A-\mathrm{MOD}\left({ }^{3}\right)$.

(2) For every A-module $N$ there is a surjection $M^{(I)} \rightarrow N$ in A-MOD for some $I$.

The definition is a direct translation of the ungraded one; the equivalent conditions for $|M|$ to be a generator in $|A|$-Mod are faithfulness of $\operatorname{Hom}_{|A|}(|M|$, on $|A|$-Mod, and the existence of a surjective arrow $|M|^{(I)} \rightarrow N$ in $|A|$-Mod for any $|A|$-module $N$.

(3.12) LEMMA. If $A=\left(A_{0}\right), M$ is a GENERATOR if and only if $|M|$ is a generator.

(Indeed when $A=\left(A_{0}\right),||$ is an equivalence of categories, $A$-MOD $\rightarrow|A|-$ Mod.)

(3.13) Lemma. Let $M$ be a projective A-module. Then $M$ is finitely generated $\Leftrightarrow \operatorname{HOM}_{A}(M$,$) preserves arbitrary coproducts.$

(3.14) Corollary and Definition. An A-module $M$ is called FAITHFUlLY projective if it satisfies the following conditions, which are equivalent:

(1) $\operatorname{HOM}_{A}(M$,$) is "faithfully exact" on A$-Mod, that is, it is faithful and exact and it preserves arbitrary coproducts.

(2) $M$ is a finitely generated projective GENERATOR.

(3.15) Corollary. Let $M$ and $L$ be $k$-modules.

(i) The following are equivalent:

(1) $M$ is FAITHFULL Y projective.

(2) $M$ is finitely generated and projective and $\operatorname{Ann}_{K}(M)=0$.

(3) $M \otimes_{k} N \approx k^{(n)} \oplus(\tau k)^{(m)}$ for some $k$-module $N, n+m>0$.

(ii) $\mathrm{END}_{k}(M)$ is FAITHFULLY projective if $M$ is.

(iii) $M \otimes_{k} L$ is FAITHFULLY projective $\Leftrightarrow M$ and $L$ are.

$\left(^{3}\right)$ Equivalently, on $A$-Mod, since $\operatorname{HOM}_{A}(X, Y)_{1}=\operatorname{Hom}_{A}(X, \tau Y)$. 
Proof. (i) In view of (3.12), (1) $\Leftrightarrow$ (2) follows from [2, II.4.8] or [3, II.5.9], and (2) $\Leftrightarrow$ (3) follows from [3, I.6.1].

(ii) and (iii) follow easily from (i).

(3.16) Lemma. Let $N$ be an $A_{k} B$-bimodule and let $M$ (resp. L) be a right $A$ - (resp. right $B$-) module. Then there is a degree-zero $k$-isomorphism

$$
\operatorname{HOM}_{A}\left(M, \operatorname{HOM}_{B}(N, L)\right) \rightarrow \operatorname{HOM}_{B}\left(M \otimes_{A} N, L\right) .
$$

It induces an isomorphism

$$
\operatorname{Hom}_{A}\left(, \mathbf{H O M}_{B}(N,)\right) \rightarrow \operatorname{Hom}_{B}\left(\otimes_{A} N, \quad\right)
$$

of $k$-functors, i.e.,

$$
\operatorname{Mod}-A \underset{\mathbf{H O M}_{B}(N,)}{\stackrel{\otimes_{A} N}{\leftrightarrows}} \operatorname{Mod}-B
$$

are adjoint.

Proof. The maps

$$
\operatorname{HOM}_{A}\left(M, \operatorname{HOM}_{B}(N, L)\right) \underset{\psi}{\stackrel{\varphi}{\rightleftarrows}} \operatorname{HOM}_{B}\left(M \otimes_{A} N, L\right)
$$

defined by $(\varphi f)(m \otimes n)=(f m) n$, and $((\psi g) m) n=g(m \otimes n)$, provide inverse isomorphisms.

Although (3.16) will not be directly in evidence below, it is useful in the proof of (3.18).

Call a $k$-functor $T: \operatorname{Mod}-A \rightarrow \operatorname{Mod}-B$ a $\tau$ - $k$-functor if $\tau T \approx T \tau$ as functors Mod- $A \rightarrow$ Mod- $B$. Any such functor may be canonically extended to a $k$-functor MOD- $A \rightarrow$ MOD- $B$ which is degree-preserving on morphisms, by means of the identifications $\operatorname{HOM}_{A}(M, N)_{1}=\operatorname{Hom}_{A}(M, \tau N)$. If $T$ and $S$ are $k$-functors Mod- $A \rightarrow$ Mod- $B$, a degree-zero natural transformation $t: T \rightarrow S$ consists of a $t_{x} \in \operatorname{Hom}_{B}(T X, S X)$ for each $A$-module $X$, such that for any $f \in \operatorname{Hom}_{A}(X, Y)$,

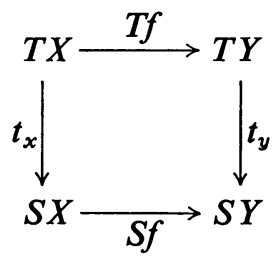

commutes. If $T$ and $S$ are $\tau$-k-functors we require also that $t$ and $\tau$ commute, in the sense that

commutes for all $X$.

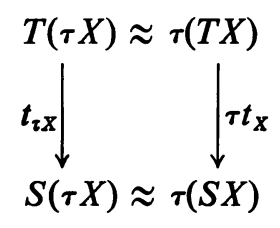


With degree-zero natural transformations as morphisms,

$$
\tau-k \text {-functors (Mod- } A \text {, Mod- } B \text { ) }
$$

is almost a category. Our chief concern will be with $\tau$ - $k$-functors of the form $\otimes_{A} M$ for some bimodule ${ }_{A} M_{B}$, and morphisms among such form a set by

(3.17) Proposition. $M \mapsto \bigotimes_{A} M$ defines a fully faithful functor

$$
G: A \otimes_{k} B^{*} \text {-Mod } \rightarrow \tau-k \text {-functors (Mod- } A \text {, Mod-B). }
$$

Let us outline the proof. One sees easily that $G$ is a functor and that the maps $\operatorname{Hom}_{A \otimes B^{*}}(M, N) \rightarrow \operatorname{Hom}\left(\otimes_{A} M, \otimes_{A} N\right)$ are always injective; it remains to show that they are surjective. Let $t$ be a degree-zero natural transformation $G M \rightarrow G N$. In particular $t_{A}$ is a right $B$-morphism $A \otimes_{A} M \rightarrow A \otimes_{A} N$, corresponding to a right $B$-morphism $f: M \rightarrow N$. Now for any $a \in A$, left multiplication by $a$ is an element $a$ of $\mathbf{H O M}_{A}(A, A)$, and therefore, by naturality, $t_{A} \circ(a \otimes M)$ $=(a \otimes N) \circ t_{A}$ (using the extensions of $G M, G N$ and $t$ to MOD- $A \rightarrow$ MOD- $B$ ). This just says that $t_{A}$, and hence also $f$, is a bimodule morphism. It is not hard to show that $t=G f$.

Call a $\tau$ - $k$-functor right continuous if it preserves arbitrary coproducts and is right exact. Again, $\otimes_{A} M$ is an example, for any bimodule ${ }_{A} M_{B}$, and these are the only examples by

(3.18) Proposition (Eilenberg, Watts). $G$ induces a bijection from isomorphism classes of $\mathrm{Ob}\left(A \otimes B^{*}\right.$-Mod) to isomorphism classes of right continuous $\tau-k$-functors Mod- $A \rightarrow$ Mod- $B$. If the bimodule ${ }_{A} M_{B}$ (resp. ${ }_{B} N_{C}$ ) corresponds to $S$ (resp. $T)$ then the bimodule ${ }_{A}\left(M \otimes_{B} N\right)_{C}$ corresponds to $T \circ S$.

The last statement is the observation that $X \otimes_{A}\left(M \otimes_{B} N\right) \approx\left(X \otimes_{A} M\right) \otimes_{B} N$ for $X \in \mathrm{Ob}$ Mod- $A$. Injectivity is the assertion that $M \approx N$ as bimodules if and only if $\otimes_{A} M \approx \otimes_{A} N$ as functors, and this follows from (3.17). The proof of surjectivity is similar to the proof of surjectivity in (3.17); given a right continuous $\tau-k$ functor $T$ : Mod- $A \rightarrow$ Mod- $B, T A$ is an $A_{k} B$-bimodule, and $T \approx \otimes_{A} T A$.

(3.19) Corollary AND Definition. Call a bimodule ${ }_{A} M_{B}$ invertible if it satisfies the following conditions, which are equivalent:

(1) $\otimes_{A} M$ is an equivalence, Mod- $A \rightarrow$ Mod- $B$.

(2) There is a bimodule ${ }_{B} N_{A}$ and degree-zero bimodule isomorphisms $M \otimes_{B} N \approx_{A} A_{A}$ and $N \otimes_{A} M \approx_{B} B_{B}$.

(3.20) Proposition. Let

$$
\operatorname{Mod}-A \underset{S}{\stackrel{T}{\rightleftarrows}} \operatorname{Mod}-B
$$

be $\tau$ - $k$-equivalences, and set $P=T A$ and $Q=S B$. Then $P$ (resp. $Q)$ is an $A_{k} B$ - (resp. $\left.B_{k} A-\right)$ bimodule, and 
(i) $T \approx \otimes_{A} P$ and $S \approx \otimes_{B} Q$.

(ii) There are degree-zero bimodule isomorphisms $f: P \otimes_{B} Q \rightarrow{ }_{A} A_{A}$ and $g: Q \otimes_{A} P \rightarrow{ }_{B} B_{B}$, and

(iii) $f$ and $g$ can be so chosen that

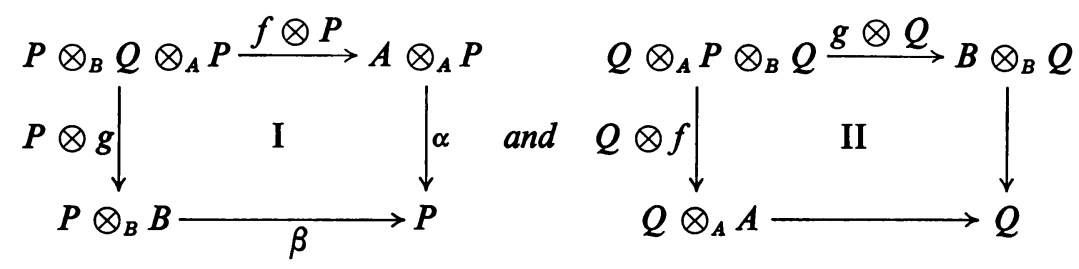

commute, where $\alpha$ and $\beta$, and their counterparts in II, are the natural isomorphisms.

(i) and (ii) are in fact contained in the statements and proofs of (3.18) and (3.19), and (iii) is proved as in the ungraded case (cf. [3, II.4.1]): given $f$ and $g$ as in (ii), we can modify $f$ by a unit of $A_{0}$ to make I commute, and II then commutes automatically.

We now abstract the situation described in (3.20).

(3.21) Definition. $A$ set of graded pre-equivalence data (GPED) over $k$ is $a$ sextuple $(A, B, P, Q, f, g)$ where $A$ and $B$ are (graded) $k$-algebras, $P($ resp. $Q)$ is an $A_{k} B$ (resp. $\left.B_{k} A\right)$ bimodule, and $f\left(\right.$ resp. $g$ ) lives in $\operatorname{Hom}_{A^{*}}\left(P \otimes_{B} Q, A\right)$ (resp. in $\left.\operatorname{Hom}_{B^{e}}\left(Q \otimes_{A} P, B\right)\right)$, provided that $(p q) p^{\prime}=p\left(q p^{\prime}\right)$ and $(q p) q^{\prime}=q\left(p q^{\prime}\right)$ for all $p, p^{\prime} \in P, q, q^{\prime} \in Q$, where $p q$ (resp. $\left.q p\right)$ abbreviates $f(p \otimes q)$ (resp. $g(q \otimes p)$ ). It is a set of graded equivalence data (GED) over $k$ if $f$ and $g$ are isomorphisms.

(3.22) THEOREM. Let $(A, B, P, Q, f, g)$ be a GPED over $k$, and assume $f$ is surjective. Then

(i) $f$ is an isomorphism.

(ii) $P$ (resp. $Q$ ) is a GENERATOR in A-MOD (resp. in MOD-A).

(iii) $P$ (resp. $Q$ ) is finitely generated and projective in Mod- $B$ (resp. in $B$-Mod).

(iv) $g$ induces degree-zero bimodule isomorphisms ${ }_{B} Q_{A} \rightarrow \operatorname{HOM}_{B}(P, B)$ and ${ }_{A} P_{B} \rightarrow \operatorname{HOM}_{B}(Q, B)$.

(v) The bimodule structures of $P$ and $Q$ induce $k$-algebra isomorphisms $\operatorname{END}_{B}(P) \leftarrow A \rightarrow \operatorname{END}_{B}(Q)^{*}$.

The proof of the ungraded analogue of this theorem, [3, II.4.3], lifts, mutatis mutandis, to prove (3.22). Capitalization of HOM and END introduces some signs, but this poses no essential difficulty.

(3.23) TheOREM. Let $(A, B, P, Q, f, g)$ be a GED over $k$. Then

(i) ${ }_{A} P_{B}$ and ${ }_{B} Q_{A}$ are invertible bimodules and

$$
\operatorname{Mod}-A \underset{\otimes_{B} Q}{\stackrel{\otimes_{A} P}{\leftrightarrows}} \text { Mod- } B
$$


and

are $k$-equivalences.

$$
A \text {-Mod } \underset{P \otimes_{B}}{\stackrel{Q \otimes_{A}}{\rightleftarrows}} B \text {-Mod }
$$

(ii) $P$ (resp. $Q$ ) is FAITHFULL Y projective in A-MOD and in MOD-B (resp. in $B$-MOD and in MOD- $A$ ).

(iii) $f$ and $g$ induce degree-zero bimodule isomorphisms

$$
\operatorname{HOM}_{B}(Q, B) \leftarrow{ }_{A} P_{B} \rightarrow \operatorname{HOM}_{A}(Q, A)
$$

and

$$
\mathbf{H O M}_{B}(P, B) \leftarrow{ }_{B} Q_{A} \rightarrow \operatorname{HOM}_{A}(P, A) .
$$

(iv) The natural maps

$$
\operatorname{END}_{B}(P) \leftarrow A \rightarrow \operatorname{END}_{B}(Q)^{*} \text { and } \operatorname{END}_{A}(Q) \leftarrow B \rightarrow \operatorname{END}_{A}(P)^{*}
$$

are $k$-algebra isomorphisms.

(v.) The equivalences of (i) induce k-algebra isomorphisms CENTER $A$ $\approx \operatorname{CENTER}_{B} \approx \operatorname{END}_{A^{e}}(A) \approx \operatorname{END}_{B^{e}}(B) \approx \operatorname{END}_{A \otimes B^{*}}(P) \approx \operatorname{END}_{B \otimes A^{*}}(Q)$.

(vi) The maps

$$
\begin{array}{ll}
\text { \{right ideals of } A\} \rightarrow \text { right } B \text {-submodules of } P\} & \mathfrak{A} \mapsto \mathfrak{A} P \\
\{\text { left ideals of } A\} \rightarrow\{\text { left } B \text {-submodules of } Q\} & \mathfrak{A} \mapsto Q \mathfrak{A} \\
\{\text { right ideals of } B\} \rightarrow\{\text { right } A \text {-submodules of } Q\} & \mathfrak{B} \mapsto \mathfrak{B Q}
\end{array}
$$

and

$$
\{\text { left ideals of } B\} \rightarrow\{\text { left } A \text {-submodules of } P\} \quad \mathfrak{B} \mapsto P \mathfrak{B}
$$

are bijective, and in each case two-sided ideals correspond to sub-bimodules. In particular the four lattices \{two-sided ideals of $A\}$, \{two-sided ideals of $B\},\left\{A_{k} B-\right.$ submodules of $P\}$ and $\left\{B_{k} A\right.$-submodules of $\left.Q\right\}$ are isomorphic. (Recall that all ideals and submodules are graded.)

Proof. (i) follows from (3.19), and (ii)-(iv) follow from (ii)-(v) of (3.22). For (v), we have CENTER $A=A^{A}=\operatorname{END}_{A^{e}}(A) \approx \operatorname{END}_{A \otimes B^{*}}(P)$ since $\otimes_{A} P$ is an equivalence, etc. For (vi), projectivity of $P$ implies that $\mathfrak{A} \otimes_{A} P \rightarrow \mathfrak{A} P$ is an isomorphism, and we can appeal again to the fact that $\otimes_{A} P$ is an equivalence, and similarly for the other cases.

Let $P$ be a right $B$-module. Guided by (3.22), we will construct a GPED from $B$ and $P$, and give a criterion, in terms of $B$ and $P$, for it to be a GED.

Put $A=\mathbf{E N D}_{B}(P)$ and $Q=\mathbf{H O M}_{B}(P, B)$. For $p \in P, q \in Q$ define $p q \in A$ by $(p q) p^{\prime}=p\left(q p^{\prime}\right) \forall p^{\prime} \in P$; this defines a map $f_{P}: P \otimes_{B} Q \rightarrow A$. Define $g_{P}: Q \otimes_{A} P \rightarrow B$ by $g(q \otimes p)=q p=q(p)$.

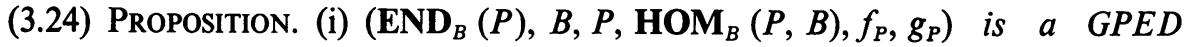
over $k$. 
(ii) $\operatorname{Im} f_{P}$ is a two-sided ideal of $A ; \operatorname{Im} f_{P}=A \Leftrightarrow f_{P}$ is an isomorphism $\Leftrightarrow P$ is $a$ finitely generated projective $B$-module.

(iii) $\operatorname{Im} g_{P}$ is a two-sided ideal of $B ; \operatorname{Im} g_{P}=B \Leftrightarrow g_{P}$ is an isomorphism $\Leftrightarrow P$ is $a$ GENERATOR in MOD-B.

(iv) The GPED of (i) is a GED $\Leftrightarrow P$ is a FAITHFULLY projective B-module.

(3.25) Corollary. A right $B$-module $P$ is projective $\Leftrightarrow \exists p_{i} \in h P, q_{i} \in h \mathbf{H O M}_{B}(P, B)$ (where $i \in I=$ some index set) with the property that for each $p \in P, q_{i} p=0$ for almost all $i$ and $\sum p_{i}\left(q_{i} p\right)=p$. When $P$ is projective we have $P \Omega=P$ where $\Omega=\operatorname{Im} g_{P} \subset B$.

4. Local and punctual criteria for separability; tower properties. A useful tool for dealing with separable algebras is localization at primes of the ground ring. To make use of this in our present context we have first to say what we mean by $S^{-1} K$ when $S$ is a multiplicative set in a commutative graded ring $K$. Some caution is required: if there are nonhomogeneous elements in $S$, for example, $S^{-1} \mathrm{~K}$ will not be graded.

Even if we agree to localize only at $S \subset h K$ we cannot imitate the ungraded definition too naïvely: $(s, x) \sim\left(s^{\prime}, x^{\prime}\right) \Leftrightarrow \exists t \in S, t\left(s^{\prime} x-s x^{\prime}\right)=0$ does not define an equivalence relation on $S \times K$, because of signs which occur when we commute elements of $K$. One way around this difficulty is to take the signs into account in the definition: $(s, x) \sim\left(s^{\prime}, x^{\prime}\right) \Leftrightarrow \exists t \in S, t\left(s^{\prime} x-(-1)^{s s^{\prime}} s x^{\prime}\right)=0$ does define an equivalence relation, and the set of classes, with the obvious structure of graded $K$ algebra, is a reasonable candidate for $S^{-1} K$. A more drastic solution would be to localize only at $S \subset K_{0}$; for such $S$ the sign problem evaporates, and indeed $S^{-1} M$ (for any graded $K$-module $M$ ) and $S^{-1} K$ are already defined (because $K_{0}$ is a commutative ring). We will see that this restricted notion of localization will suffice for our purposes.

As usual call an ideal $m$ of $K$ maximal if $m \neq K$ and the only ideal properly containing $m$ is $K$ itself. (Recall that ideal always means graded ideal.) Equivalently, $m$ is maximal if $K / m$ is a simple graded ring, i.e., it has no proper (graded) ideals. If $m$ is a maximal ideal of $K, S_{m}=h(K-m)$ is a multiplicative subset, and $S_{m}^{-1} K$, defined as above, is a reasonable candidate for $K_{m}$. The more drastic solution indicated above would suggest looking instead at the localization with respect to $h(K-m) \cap K_{0}=K_{0}-m_{0}$. This is less absurd than it seems at first glance, because of

(4.1) LEMMA. $m \mapsto m_{0}=m \cap K_{0}$ is a bijection, \{maximal ideals of $\left.K\right\} \rightarrow\{$ maximal ideals of $\left.K_{0}\right\}$.

According to (4.1), the more drastic alternative amounts simply to localizing at maximal ideals of $K_{0}$ instead of those of $K$.

(4.1) may be proved directly, or a proof can be extracted from the following two propositions, which are also useful independent of (4.1).

(4.2) Proposition. If $K_{1}$ is $K_{0}$-free, either $K_{1} K_{1}=0$ or $K=K_{0}\langle a\rangle, a \neq 0$. In particular, $|K|$ is commutative. 
(4.3) Proposition. If $K$ is simple then $K_{0}$ is a field and either $K_{1}=0$ or $K=K_{0}\langle a\rangle, a \neq 0$, and Char. $K=2$ in the second case.

Proof of (4.2). If $\alpha, \beta \in K_{1}$ are distinct basis elements, $\partial(\alpha \beta)=0$, and hence $(\alpha \alpha) \beta=(\alpha \beta) \alpha$, so that $\alpha \alpha=\alpha \beta=0$ by linear independence. Similarly $\beta \beta=\beta \alpha=0$. This shows that if $K_{1} K_{1} \neq 0, K_{1}$ is free of rank one over $K_{0}$, and we can write $K_{1}=K_{0} t$. Clearly $a=t^{2} \neq 0$ (otherwise $K_{1} K_{1}=0$ ), and obviously $\left|K_{0}\langle a\rangle\right|$ is commutative.

Proof of (4.3). If $0 \neq t \in K_{0}, K t$ is a nonzero ideal, hence $K t=K$. Thus $K_{0}$ is a field and we have the conclusions of (4.2). If $K_{1} K_{1}=0, K_{1}$ is an ideal, and therefore $K_{1}=0$. Whenever $K$ and $|K|$ are both commutative we have $2 x y=0$ for all $x, y \in K_{1}$, for $x y=y x=-x y$. When $K_{1} \neq 0, K_{1} K_{1}$ contains the nonzero element $a$, and since $K_{0}$ is a field this implies Char. $K=2$.

The reader will notice that these arguments do not require commutativity of $K$. For " $(\alpha \alpha) \beta=(\alpha \beta) \alpha$ " we need $K_{0} \subset$ CENTER $K$, but commutativity in the $K_{1}$ part is used nowhere except in the last statement ("Char. $K=2$ ") of (4.3). Call $K$ quasicommutative if $K_{0} \subset$ CENTER $K$; then (4.2) and (4.3) hold for quasi-commutative $K$, except for the statement about the characteristic in (4.3). (4.1) also holds for quasi-commutative $K$, if "ideal of $K$ " is interpreted to mean "two-sided ideal of $K$ ".

As an immediate corollary of (4.1), we have

(4.4) Proposition. Let $A$ be a K-algebra, finitely presented as a $K$-or $A_{K}^{e}$-module. The following are equivalent:

(1) $A$ is K-separable.

(2) $A_{m}$ is $K_{m}$-separable for every maximal ideal $m$ of $K_{0}$.

(3) $A_{m_{0}}$ is $K_{m_{0}}$-separable for every maximal ideal $m$ of $K$.

Proof. (2) $\Leftrightarrow$ (3) by (4.1), (1) $\Rightarrow(2)$ by (3.8.ii), and (2) $\Rightarrow$ (1) by (3.10), applied to $L=\prod_{m} K_{m}$.

Further justification for the adequacy of the more restricted notion of localization in our context is provided by two versions of Nakayama's lemma:

(4.5) Lemma. If $M$ is a finitely generated (graded) $K$-module and $J \subset \operatorname{rad} K_{0}$, $J M=M \Rightarrow M=0$.

(Here rad denotes Jacobson radical: intersection of all maximal ideals.)

(4.6) Lemмa. Let $M$ be a finitely generated (graded) $K$-module where $K_{0}$ is local and let $m$ be the unique maximal ideal of $K$. Then $m M=M \Rightarrow M=0$.

(4.5) and (4.6) are easy consequences of the usual Nakayama lemma.

(4.7) Proposition. Let $P$ be a finitely generated projective $K$-module and assume $K_{0}$ is local. Then $P$ is free.

Proof. It suffices to prove this when $K_{0}$ is a field, by (4.5), and (4.2) breaks the proof in two cases. If $K_{1} K_{1}=0, K_{1}$ is an ideal, and in fact it is easy to see that nonhomogeneous elements are invertible, so that $|K|$ is a (commutative) local ring 
with maximal ideal $K_{1}$. In this situation we can apply the ungraded analogue of (4.7). If $K_{1}=K_{0} t, 0 \neq t^{2} \in K_{0}, P$ is free of finite rank as a $K_{0}$-module, say $P_{0} \approx K_{0}^{(n)}$, $P_{1} \approx K_{0}^{(m)}$, and $n=m$, for multiplication by $t$ is a $K_{0}$-isomorphism $P_{1} \rightarrow P_{0}$. It is now an easy matter to show that $P$ is isomorphic to the free $K$-module $K^{(r)} \oplus[\tau K]^{(s)}$ where $(r, s)=(n / 2, n / 2)$ if $n$ is even and $((n+1) / 2,(n-1) / 2)$ if $n$ is odd.

(4.8) Proposition. Let $P \neq 0$ be a finitely generated projective $K$-module and let $A=\operatorname{END}_{K}(P)$. Then $A$ is a separable $K$-algebra and CENTER $A \approx K / \operatorname{Ann}_{K}(P)$.

Proof. We can use (4.4) to localize, and then use (4.7) to interpret $A$ as a graded matrix algebra; the proof can then be finished by imitating the proof in the ungraded case, [3, III.2.13]. Rather than carry this out, we give a proof based on the Morita theory of $\S 3$, and valid in the case $K=\left(K_{0}\right)$. In this case $P$ is a FAITHFULLY projective module over $\widetilde{K}=K / \operatorname{Ann}_{K}(P)$, and the resulting GED $\left(A=\operatorname{END}_{K}(P)\right.$ $\left.=\operatorname{END}_{\tilde{K}}(P), \tilde{K}, P, Q=\operatorname{HOM}_{\tilde{K}}(P, \tilde{K}), f_{P}, g_{P}\right)$ yields immediately the isomorphism CENTER $A \approx$ CENTER $\tilde{K}=\tilde{K}$. For separability, we have that $P$ (resp. $Q$ ) is FAITHFULLY projective in $A$-MOD (resp. in MOD- $A=A^{*}$-MOD), so that $A \approx P \otimes_{\tilde{K}} Q$ is $A \otimes_{\tilde{K}} A^{*}$-projective. This shows that $A$ is separable over its CENTER, $\tilde{K} . \tilde{K}$ is $K$-separable by (2.10), and, anticipating (4.13(i)), we can conclude that $A$ is $K$-separable.

Our next goal is a punctual theorem, of the form " $A$ is $K$-separable $\Leftrightarrow A / m A$ is $K / m$-separable for all $m$." First, a lemma:

(4.9) Lemma. Let $M$ be an A-module, A a K-algebra; let $f \in \operatorname{End}_{A}(M)$ and assume either

(i) $M$ is Noetherian (a.c.c. on (graded) submodules), or

(ii) $|A|$ is commutative and $M$ is finitely generated.

Then if $f$ is surjective it is an isomorphism.

Proof. Case (i). Since $f$ is homogeneous (of degree zero), $\operatorname{ker}\left(f^{i}\right)$ is a submodule for each $i \geqq 1$. If $0 \neq y \in \operatorname{ker} f$, any $x$ such that $f(x)=y$ satisfies $x \in \operatorname{ker}\left(f^{2}\right)-\operatorname{ker} f$. By induction if $y \in \operatorname{ker}\left(f^{i}\right)-\operatorname{ker}\left(f^{i-1}\right)$, any $x$ such that $f(x)=y$ satisfies $x \in \operatorname{ker}\left(f^{i+1}\right)-\operatorname{ker}\left(f^{i}\right)$.

In case (ii), apply [2, IV.5.3] to $f \in \operatorname{End}_{|A|}(|M|)$.

Note that hypothesis (ii) applies in two important cases: when $A=K=\left(K_{0}\right)$, and (by (4.2)) when $A=K$ and $K_{0}$ is a field.

(4.10) Theorem. Let A be a K-algebra, and assume either

(i) $|K|$ is commutative and $A$ is finitely $K$-presented, or

(ii) $K$ is Noetherian and $A$ is a finitely generated $K$-module.

Then $A$ is $K$-separable $\Leftrightarrow A / m A$ is $K / m$-separable for every maximal ideal $m$ of $K$.

The important case of (4.10) is when $K=\left(K_{0}\right)$, and in this case the hypothesis is simply that $A$ be finitely $K$-presented, as in (4.4). (4.4) shows that (4.10) (as stated, i.e., for arbitrary $K$ ) follows from 
(4.11) Proposition. Let $K$ be commutative with $K_{0}$ local and let $A$ be a $K$-algebra, finitely generated as $K$-module. Assume either that $|K|$ is commutative or that $K$ is Noetherian. Then $A$ is $K$-separable $\Leftrightarrow A / m A$ is $K / m$-separable, where $m$ is the unique maximal ideal of $K$.

Proof of (4.11). $\Rightarrow$ follows from (3.8(ii)). For the converse, let ' denote the functor, reduction $\bmod m$ (i.e., ' $=K / m \bigotimes_{K}$ ). Then $\delta \in \operatorname{Der}_{K}(A, J)$ induces an inner derivation $\delta^{\prime}: A^{\prime} \rightarrow J^{\prime}$, say $\delta^{\prime}=d_{e^{\prime}}, e^{\prime} \in J^{\prime}$. $\partial \delta^{\prime}=0$ implies $\partial e^{\prime}=0$, so that $\delta^{\prime}\left(a^{\prime}\right)$ $=\delta^{\prime}\left(a^{\prime}\right) e^{\prime}$ for all $a^{\prime} \in A^{\prime}$. Choose pre-images $a \in A$ and $e \in J_{0}$ for $a^{\prime}$ and $e^{\prime}$, respectively; then $\delta(a) \equiv(\delta \dot{a}) e \bmod m J$, and, since $\operatorname{Im} \delta$ generates $J, J=J e+m J$. With (4.6), this implies $J=J e$ : right multiplication by $e$ is surjective on $J$. But then (4.9) implies it is an automorphism of $J$, and since it also defines an $A^{e}$-morphism, $A^{e} \rightarrow J$, we can use it to split the inclusion $J \rightarrow A^{e}$. Thus $E(A)$ splits, and $A$ is $K$-separable.

To close this section we record some "tower" or "transitivity" properties of separability.

(4.12) Lemma. Let $A$ and $B$ be $K$-algebras and assume that $A$ is $K$-projective and that $K$ is a direct summand of $A$. Then if $A \otimes_{K} B$ is $K$-separable, so is $B$.

The simple proof is a direct imitation of [1, Proposition (1.7)].

(4.13) Proposition. Let $L$ be a commutative $K$-algebra and let $A$ be an L-algebra.

(i) If $A$ is $L$-separable and $L$ is $K$-separable, $A$ is $K$-separable $\left(^{4}\right)$.

(ii) If $A$ is $K$-separable, $A$ is $L$-separable.

(iii) If $A$ is $K$-separable and $L$-projective and contains $L$ as a direct summand, $L$ is K-separable.

Proof. (i) Since $L$ is $K$-separable, $E_{K}(L)$ is split exact. Therefore $A_{K}^{e} \otimes_{L^{e}} E_{K}(L)$ is split exact, so that $A_{K}^{e} \otimes_{L^{e}} L$ is $A_{K}^{e}$-projective. But clearly $A_{K}^{e} \otimes_{L^{e}} L$ and $A_{L}^{e}$ are isomorphic $A_{K}^{e}$-modules. Thus $A_{L}^{e}$ is $A_{K}^{e}$-projective, and since $A$ is $A_{L}^{e}$-projective by hypothesis, $A$ is $A_{K}^{e}$-projective, i.e. $A$ is $K$-separable.

(ii) is trivial since $A_{L}^{e}$ is a quotient of $A_{K}^{e}$.

(iii) If $A$ is $L$-projective, $A_{K}^{e}$ is $L_{K}^{e}$-projective, and since $A$ is $K$-separable this implies $A$ is $L_{K}^{e}$-projective. Since $L$ is an $L^{e}$-direct summand of $A$, our result follows.

(4.14) Lemma. Let * denote the functor $\operatorname{Hom}_{K}(, K)$ and suppose $f \in \operatorname{Hom}_{K}(Q, M)$ with $Q$ finitely generated and projective. Then $f$ has a left inverse $\Leftrightarrow f^{*}$ is surjective. If $M$ is finitely presented, f has a left inverse $\Leftrightarrow f_{m_{0}}$ has a left inverse for every maximal ideal $m$ of $K$.

The proof follows the ungraded pattern [3, III.2.16].

Call a $K$-module $M$ faithful if $\operatorname{Ann}_{K}(M)=0 . M$ is always a faithful $\operatorname{END}_{K}(M)$ module; indeed, a $K$-module structure for $M$ is a $K$-algebra homomorphism $\psi: K \rightarrow \operatorname{END}_{K}(M)$, and $\operatorname{ker} \psi=\operatorname{Ann}_{K}(M)$. Call $M$ f-projective if it is finitely

(4) In particular if $A$ is separable over a quotient of $K$, it is $K$-separable; cf. (2.11). 
generated, projective, and faithful( $\left(^{5}\right)$. If $A$ is a $K$-algebra, the module structure $K \rightarrow \operatorname{END}_{K}(A)$ factors through $K \rightarrow A^{A}=\operatorname{END}_{A^{e}}(A) \subset \operatorname{END}_{K}(A)$, so that $\operatorname{Ann}_{K}(A)=\operatorname{ker}(K \rightarrow A)$. Thus, if $A$ is faithful, we can assume $K \subset A$. In fact,

(4.15) Lemma. If a $K$-algebra $A$ is $f$-projective, $K$ is a direct summand of $A$.

This is an immediate corollary of (4.14).

(4.16) Corollary. (i) Let $A$ and $B$ be $K$-algebras and assume $A$ is f-projective. Then if $A \otimes_{K} B$ is $K$-separable, so is $B$.

(ii) Let $L$ be a commutative $K$-algebra, $A$ an L-algebra. If $A$ is $K$-separable and $f$-projective as an L-module, $L$ is $K$-separable.

(4.17) Corollary. Let $A$ be $a$ K-algebra and set $C=$ CENTER $A$. Assume $A$ is $C$-projective. Then $A$ is $K$-separable $\Leftrightarrow A$ is $C$-separable and $C$ is $K$-separable.

REMARK. The hypothesis in (4.17), that $A$ be $C$-projective, is satisfied automatically in one important case: when $C$ is concentrated in degree zero and $A$ is finitely generated as $C$-module, $C$-separability of $A$ implies $C$-projectivity of $A$. This will be a by-product of the implication (i) $\Rightarrow$ (iii) in Theorem (6.1) below.

5. Structure theorems. The main theorem of this section, (5.5), describes the structure of simple algebras, and is due to Wall [9].

Throughout this section, ideal means two-sided ideal unless otherwise indicated. We call a ring $A$ simple if 0 and $A$ are the only ideals; if $A$ is graded this means 0 and $A$ are the only graded ideals.

In the two lemmas that follow, $A$ denotes a simple graded ring, and we fix the following notations: $K=\operatorname{CENTER} A, k=K_{0}=$ Center $A, Z=$ Center $|A|$, and $L=A^{A_{0}}=|A|^{A_{0}}$. Then we have

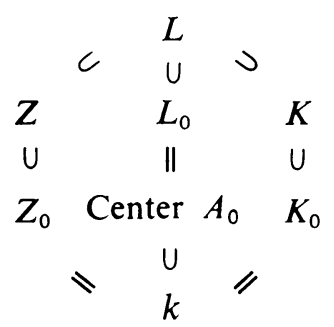

(5.2) LEMMA. $k$ is a field and $K$ and $Z$ are simple graded rings.

Proof. If $t$ is a nonzero element of $h K$ or $h Z, A t$ is a nonzero ideal, hence $A t=A$ and $t$ is invertible in $A$; the given results follow.

The next lemma relates simplicity of $A$ to simplicity of $|A|$ and $A_{0}$. We omit the proof, for which see [9, pp. 188-189].

(5) The usual term, faithfully projective, would lead to confusion; cf. (3.14) above. We have seen (in (3.15(i))) that when $K=\left(K_{0}\right), M$ is $f$-projective $\Leftrightarrow M$ is FAITHFULLY projective. 
(5.3) Lemma. Assume $A_{1} \neq 0$. Then

(i) $A_{1} A_{1}=A_{0}$, and if $0 \neq \mathfrak{A}$ is an ideal of $A_{0}$, then $A_{1} \mathfrak{A}+\mathfrak{A} A_{1}=A_{1}$ and $\mathfrak{A}+$ $A_{1} \mathfrak{A} A_{1}=A_{0}$.

(ii) If $B$ is a proper ideal of $|A|$, both projections $B \rightarrow A_{i}$ are $A_{0}$-module isomorphisms.

(iii) If $|A|$ is not simple, $A_{0}$ is a central simple $k$-algebra and $A=\left(A_{0}\right) \otimes k\langle 1\rangle$.

(iv) Either $|A|$ or $A_{0}$ is a central simple $k$-algebra, and, provided $[A: k]<\infty$, they are not both central simple.

(5.4) Lemma. Let $D$ be a central division algebra over $k$, let $F \supset k$ be a quadratic field extension $([F: k]=2)$, and set $D^{\prime}=F \otimes_{k} D$. Then $D^{\prime}$ is not a division algebra $\Leftrightarrow$ there is an imbedding of $F$ in $D$.

Proof. $D^{\prime}$ is a central simple $F$-algebra, so that $D^{\prime} \approx \mathbf{M}_{r}(E)$, the algebra of $r \times r$ matrices over $E$, for some central $F$-division algebra $E$. Choose a simple $D^{\prime}$ module $S$ (e.g., a minimal right ideal). Then $[S: k]=\left[D^{\prime}: k\right] / r=2[D: k] / r$, and since $S$ is also a $D$-module, $r=1$ or $2 . D^{\prime}$ is not a division algebra $\Leftrightarrow r=2$, in which case $[S: D]=[S: k] /[D: k]=1$. But when $S$ is a one-dimensional $D$-module we can identify $\operatorname{End}_{D}(S)$ with $D^{\circ}$, and $F=F \otimes 1 \subset D^{\prime}$ induces an imbedding

$$
F^{\circ} \rightarrow \operatorname{End}_{D^{\prime}}(S) \subset \operatorname{End}_{D}(S)=D^{\circ} .
$$

(5.5) THeOREM (WALL). Let $A$ be a simple graded ring, let $k, K, Z$ and $L$ be as in (5.1), and assume $[A: k]<\infty$. Then

Case (0). If $A_{1}=0, k=L$ and $|A|=A_{0}$ is a central simple $k$-algebra.

Case (1). If $Z_{1} \neq 0, A_{0}$ is a central simple $k$-algebra and $\exists 0 \neq a \in k$ with $Z=L$ $=k\langle a\rangle$ and $A=\left(A_{0}\right) \otimes k\langle a\rangle$.

Case (2). If $Z_{1}=0$ but $A_{1} \neq 0,|A|$ is a central simple $k$-algebra. There is an $a \in k$ such that $L=|k\langle a\rangle| \subset A_{0}$ if Char. $k \neq 2$, and $L=k\{a\} \subset A_{0}$ if Char. $k=2 ; a=0$ can occur in case (i) below when Char. $k=2$, but is ruled out in all other cases. There is a $k$-vector space $W$ and a central $k$-division algebra $D$ (both finite-dimensional) such that one of the following occurs:

(i) $W_{0} \neq 0 \neq W_{1}, D_{1}=0$, and $A=\operatorname{END}_{k}(W) \otimes_{k}(D)$.

(ii) $W_{1}=0, D_{1} \neq 0$, and $A=\left(\operatorname{End}_{k}(W)\right) \otimes_{k} D$.

(iii) $W_{1}=0=D_{1}$, and $A=\left(\operatorname{End}_{k}(W)\right) \otimes_{k}(D) \otimes_{k} \Subset$, where

$$
\begin{array}{ll}
\mathbb{E}=\left(\frac{1,-a}{k}\right) & \text { if Char. } k \neq 2, \\
\mathbb{E}=\left[\frac{1, a}{k}\right] & \text { if Char. } k=2 .
\end{array}
$$

(5.6) Corollary. Let $A$ be a simple graded ring and put $L=A^{A_{0}}$ and $k=\left[A^{A}\right]_{0}$. Then, unless $A_{1}=0, \operatorname{dim}_{k}(L)=2$.

Proof of (5.5). The conclusions in Case (0) are clear. Suppose then that $0 \neq t \in Z_{1}$. Since $A t$ is a nonzero ideal, $A_{0}=A_{1} t$ and $A_{1}=A_{0} t$, so that $A=A_{0} \otimes k\langle a\rangle$ where 
$a=t^{2}$. $\left(a \neq 0\right.$, for otherwise $0=A_{1} A_{1}=A_{0}$ by (5.3(i)).) Clearly this yields $Z=L$ $=k\langle a\rangle . A_{1}=A_{0} t$ implies Center $A_{0} \subset Z_{0}=k$, so $A_{0}$ is central over $k$. Finally, if $0 \neq \Omega$ is an ideal of $A_{0}, A_{1} \Omega A_{1}=A_{0} \Omega t^{2}=\Omega$, and, by (5.3(i)), $\Omega=A_{0}$. This settles Case (1).

If $Z_{1}=0$ (but $A_{1} \neq 0$ ), $|A|$ is central over $Z_{0}=k .|A|$ is simple too, for if not the degree-one generator of $k\langle 1\rangle$ in (5.3(iii)) is a nonzero element of $Z_{1}$. Thus $|A|$ $\approx \mathbf{M}_{n}(D)$ for some central $k$-division algebra $D$. It remains to determine as explicitly as possible the graded structure of $A$, and to find $L$. The argument breaks into several cases.

First assume Char. $k \neq 2$. Define $T:|A| \rightarrow|A|$ by $T\left(a_{0}+a_{1}\right)=a_{0}-a_{1} . T$ is inner by Skolem-Noether: there is a unit $u \in|A|$ with $T(x)=u x u^{-1}$ for all $x \in|A|$. Since $T(u)=u$ we have $u \in A_{0}$, but $u \notin k$ since $T$ is not the identity. However $a=u^{2} \in k$ (since $T^{2}$ is the identity), and $a \neq 0$ because $u$ is invertible. By definition of $T$, $A_{0}=|A|^{\{u\}}=|A|^{k[u]}$ and $A_{1}=\{x \in A \mid x u=-u x\}$. We "compute" the graded structure of $A$ by choosing a representation $|A| \approx \mathbf{M}_{n}(D)$ in which $u$ has a convenient form.

Assume that $k[u]$ is not a field, so that $u^{2}=a=b^{2}, 0 \neq b \in k$. We can then assume $a=1$ by replacing $u$ with $u / b$. Then put $e=(1-u) / 2$. Then $e^{2}=e$, and the right $|A|-$ module $e|A|$ is a direct sum of, say, $r$ copies, of the simple right $|A|$-module, $D^{(n)}$. Thus $\operatorname{dim}_{k} e|A|=r n[D: k]$ and $r<n$. Let $e^{\prime}=\left(\begin{array}{cc}I_{r} & 0 \\ 0 & 0\end{array}\right) \in \mathbf{M}_{n}(D)$, then $\operatorname{dim}_{k} e|A|$ $=\operatorname{dim}_{k} e^{\prime}|A|$, and a Skolem-Noether argument yields the existence of an inner automorphism which interchanges $e$ and $e^{\prime}$. Thus assume our basis in $\mathbf{M}_{n}(D)$ chosen so that $e^{\prime}=e$; then $u=1-2 e=\left(\begin{array}{cc}-I_{r} & 0 \\ 0 & I_{n-r}\end{array}\right)$, and $A_{0}=|A|^{\{u\}}=\left\{\left(\begin{array}{cc}* & 0 \\ 0 & *\end{array}\right)\right\}$ and $A_{1}=\{x \mid x u=-u x\}=\left\{\left(\begin{array}{cc}0 & * \\ * & 0\end{array}\right)\right\}$. We are in case (i), with $\operatorname{dim}_{k}\left(W_{0}\right)=r, \operatorname{dim}_{k}\left(W_{1}\right)=n-r$.

If $k[u]$ is a field, $A_{0}$ is simple and $k[u]=L=$ Center $A_{0}$ (e.g., by [7, Theorem 9]), and (5.4) distinguishes two subcases. If $k[u]$ imbeds in $D$, we can choose a basis in which $k[u] \subset D$ (by a Skolem-Noether argument). Then $D$ is an invariant subspace for $T$, and so inherits a grading, and $|A|=\left(\mathbf{M}_{n}(k)\right) \otimes_{k} D$ as graded algebras. We are in case (ii), with $\operatorname{dim}_{k}(W)=n$.

If $D^{\prime}=L \otimes_{k} D$ is a central L-division algebra, we claim first that $n$ is even. Since $\left[D^{\prime}: D\right]=2$, this follows if $D^{(n)}$ admits a $D^{\prime}$-module structure. Now in the diagram

$$
\begin{array}{cc}
|A|=\operatorname{End}_{D^{\circ}}\left(D^{\circ(n)}\right) \subset \operatorname{End}_{k}\left(D^{\circ(n)}\right)=E, \\
U & U \\
L & D
\end{array}
$$

$|A|$ is clearly the centralizer, in $E$, of $D$. In particular, the images of the inclusions $D \rightarrow E$ and $L \rightarrow E$ commute, and therefore the inclusions induce a map $D^{\prime}=L \otimes D \rightarrow E$, thereby giving $D^{\circ(n)}$ a $D^{\prime}$-module structure.

Hence $n$ is even, say $n=2 m$. Put $u_{1}=\operatorname{diag}_{m}(\bar{u}) \in \mathbf{M}_{n}(k)$, where $\bar{u}=\left(\begin{array}{ll}0 & 1 \\ a & 0\end{array}\right)$, and $a=u^{2}$ as before. Since $u_{1}^{2}=a, k[u]$ and $k\left[u_{1}\right]$ are isomorphic quadratic field extensions of $k$, and a Skolem-Noether argument allows us to assume $u=u_{1}$ by a suitable choice of basis in $\mathbf{M}_{n}(D)$. Then $A=\left(\mathbf{M}_{m}(D)\right) \otimes \mathbf{M}_{2}(k)$, with the grading 
in $\mathbf{M}_{2}(k)$ induced by $\bar{u}$. Now $\left(\begin{array}{ll}w & x \\ y & z\end{array}\right)$ commutes with $\bar{u} \Leftrightarrow w=z$ and $y=a x$, and anticommutes $\Leftrightarrow w=-z$ and $y=-a x$. Let $s=\left(\begin{array}{rr}-1 & 0 \\ 0 & 1\end{array}\right)$ and $t=\left(\begin{array}{cc}0 & 1 \\ -a & 0\end{array}\right)$, then the degree zero term of $\mathbf{M}_{2}(k)$ is $|k \oplus k \bar{u}|$ and the degree one term is $|k s \oplus k t|$. Since $s^{2}=1$, $t^{2}=-a$, and $s t=-\bar{u}=-t s$, this establishes an isomorphism

$$
\mathbf{M}_{2}(k) \approx\left(\frac{1,-a}{k}\right)
$$

and we are in case (ii).

We have also seen that $L=|k\langle a\rangle| \subset A_{0}$ when Char. $k \neq 2$, except possibly when $k[u]$ was not a field, that is, in case (i). But, in any case, $A_{0}=|A|^{k[u]}$ implies $k[u] \subset L$, and, with $L=|A|^{A_{0}}$, we also get $L \subset k[u]$.

This completes the proof of (5.5) and (5.6) except in Case (2) when Char. $k=2$. We still have $|A| \approx \mathbf{M}_{n}(D)$, and the strategy is the same: determine the graded structure of $A$ by a convenient choice of basis for $\mathbf{M}_{n}(D)$ in the various subcases.

In characteristic two, the projection $p:|A| \rightarrow A_{1}, p\left(a_{0}+a_{1}\right)=a_{1}$, is a derivation: $p\left(\left(a_{0}+a_{1}\right)\left(b_{0}+b_{1}\right)\right)=a_{1} b_{0}+a_{0} b_{1}=a_{1} b+a b_{1}$ because $2 a_{1} b_{1}=0 . p$ is inner by $(2.10)$ (apply (2.10(5)) to the $k$-algebra $|A|): \exists u \in|A|$ with $x_{1}=x u+u x$ for all $x \in|A|$. By definition of $p, A_{0}=|A|^{\{u\}}=|A|^{k[u]}$ and $A_{1}=\{x \in A \mid x=x u+u x\}$. Thus $u \in A_{0}$ and $u \notin k$. Since $p^{2}=p, x u^{2}+u^{2} x=p x=x u+u x$ for all $x$, hence $a=u^{2}+u \in k$. As in the Char. $\neq 2$ case, this description of $A_{0}$ implies $L=k[u]=k\{a\} \subset A_{0}$.

As before, consider first the case where $k[u]$ is not a field: $\wp(u)=a=\wp(b)$, $b \in k\left({ }^{6}\right)$. Let $e=u+b$, then $e^{2}=e$. Put $e_{1}=\left(\begin{array}{cc}I_{r} & 0 \\ 0 & 0\end{array}\right) \in \mathbf{M}_{n}(k)$ with $r<n$ computed from $e$, as before, by the requirement $\operatorname{dim}_{k} e|A|=r n[D: k]$. As before we can assume that $e=e_{1}$, hence also that

$$
u=e_{1}+b=\left(\begin{array}{cc}
I_{r}+b I_{r} & 0 \\
0 & b I_{n-r}
\end{array}\right)
$$

It is easy to check that this puts us in case (i).

If $k[u]$ is a field which imbeds in $D$, we are in Case (ii) by the argument already given in the Char. $k \neq 2$ case. If $D^{\prime}=L \otimes D$ is a central $L$-division algebra, $n=2 m$ as before, and we put $u_{1}=\operatorname{diag}_{m}(\bar{u}) \in \mathbf{M}_{n}(k)$, where $\bar{u}=\left(\begin{array}{cc}0 & 1 \\ a & 1\end{array}\right) \in \mathbf{M}_{2}(k)$, and $0 \neq a$ $=\wp(u)$. As before we can arrange $u=u_{1}$ (because $\wp\left(u_{1}\right)=a$ ), and

$$
A=\left(\mathbf{M}_{m}(D)\right) \otimes \mathbf{M}_{2}(k)
$$

with grading in $\mathbf{M}_{2}(k)$ given by $\bar{u}$. Now $c=\left(\begin{array}{ll}w & x \\ y & z\end{array}\right)$ commutes with $\bar{u} \Leftrightarrow x a=y$ and $z=w+x$, and $c=c \bar{u}+\bar{u} c \Leftrightarrow x=w a+y=z$. Let $s=\left(\begin{array}{ll}1 & 0 \\ 1 & 1\end{array}\right)$ and $t=\left(\begin{array}{ll}0 & 1 \\ a & 0\end{array}\right)$, then the degree zero term of $\mathbf{M}_{2}(k)$ is $|k \oplus k \bar{u}|$ and the degree one term is $|k s \oplus k t|$. Since $s^{2}=1, t^{2}=a, s t=\bar{u}$ and $s t+t s=\bar{u}+\left(\begin{array}{ll}1 & 1 \\ a & 0\end{array}\right)=1$, we have an isomorphism

$$
\mathbf{M}_{2}(k) \approx\left[\frac{1, a}{k}\right]
$$

and we are in case (iii). This completes the proof of (5.5)-(5.6).

$\left.{ }^{(}\right)$Here $\varphi(X)=X^{2}+X$. We are in this first case, for example, when $a=0$; $u$ is then idempotent, so that $k[u]=k\{0\} \approx k \times k$. 
Using (5.5) we can get structural characterizations of separable algebras. We start with some lemmas.

Let $A$ be a graded $K$-algebra. We say $A$-MOD is semisimple if every (left) $A$ module is projective.

(5.7) Lemma. (i) If $A$ is simple, $A$-MOD is semisimple. If $K$ is simple, every $K$ module is actually free.

(ii) If $K$ is simple and $A$ is $K$-separable, $A^{e}$-MOD is semisimple.

Proof. (i) If $M$ is an $A$-module, there is a surjection $F \rightarrow M$ with $F$ free. $F$ is a direct sum of simple modules (viz. $A$ and $\tau A$ ), and, by a standard argument (cf. for example [2, III.1.1]) it follows that every submodule is a direct summand. In particular, the kernel of $F \rightarrow M$ is a direct summand, so $M$ is projective. The stronger result in the commutative case was essentially noted in the proof of (4.7).

(ii) Since separability of $A$ implies separability of $A^{e}$ it suffices to show $\operatorname{HOM}_{A}(M$,$) is exact for every M$, and since $\operatorname{HOM}_{A^{e}}(A$,$) is exact, this follows$ from

(5.8) LemMA. There is a degree zero natural isomorphism

$$
\mathrm{HOM}_{A^{e}}\left(A, \mathrm{HOM}_{K}(,)\right) \rightarrow \operatorname{HOM}_{A}(,)
$$

of functors $A$-Mod ${ }^{\circ} \times A$-MOD $\rightarrow K$-MOD.

Proof. For $A$-modules $M$ and $N$, make $\operatorname{HOM}_{K}(M, N)$ an $A^{e}$-module by $((a \otimes b) f) x=-1^{b f} a(f(b x))$. Then

$$
\operatorname{HOM}_{A^{e}}\left(A, \operatorname{HOM}_{K}(M, N)\right) \underset{\psi}{\stackrel{\varphi}{\rightleftarrows}} \operatorname{HOM}_{A}(M, N)
$$

defined by $\varphi f=f(1)$ and $((\psi g) a) x=g(a x)(a \in A, x \in M)$ are the required inverse isomorphisms.

(5.9) LemMA. Let $A$ be a simple graded ring, finitely generated as a module over $k=C_{0}, C=A^{A}$. View $A$ as a $k$-algebra and let $B$ be any ungraded $k$-algebra. Then every two-sided ideal of $A \otimes_{k}(B)$ is of the form $A \otimes_{k}(J)$ for a two-sided ideal $J$ of $B$.

Proof. Use (5.5) to distinguish two cases: either

(1) $|A|$ is a central simple $k$-algebra; or

(2) $A=\left(A_{0}\right) \otimes k\langle a\rangle, 0 \neq a \in k$, and $A_{0}$ is a central simple $k$-algebra.

In Case (1) we apply the ungraded analogue [3, III.3.4]. In Case (2), if $I$ is an ideal of $A \otimes(B)=\left(A_{0} \otimes B\right) \otimes k\langle a\rangle, I=\left(I_{0}\right) \otimes k\langle a\rangle$. But, by the ungraded case, $I_{0}=A_{0} \otimes($ an ideal of $B)$.

(5.10) Lemma. Let $K$ be a simple commutative graded ring and let $C$ be a simple commutative $K$-algebra, finitely generated as $K$-module. Assume $C$ is $K$-separable. Then $C_{0}$ is $K_{0}$-separable, and $C=\left(C_{0}\right) \otimes_{K_{0}} K$. 
Proof. We will obtain the first statement, $C_{0}$ is $K_{0}$-separable, in proving the implication (1) $\Rightarrow(2)$ of (5.11). For the second part, $C=\left(C_{0}\right) \otimes_{K_{0}} K$, it suffices to see that $C=C_{0}\langle 1\rangle$ cannot be $K=\left(K_{0}\right)$-separable. $|C|=\left|C_{0}\langle 1\rangle\right|$ is the group-ring over $C_{0}$ of a group of order two, and $\left|C^{e}\right|=\left|C \otimes_{K_{0}} C\right|=|C| \otimes_{K_{0}}|C|$ is therefore the group-ring over $C_{0}$ of a group of order four. $(|C \otimes C|=|C| \otimes|C|$ because $C$ has characteristic two.) Now according to [8, IX, $§ 1$, Corollary to Theorem 2], the radical of $\left|C^{e}\right|$ is a maximal ideal. Thus $\left|C^{e}\right|$ is a (commutative) local ring. Since $|C|$ is visibly not $\left|C^{e}\right|$-free, it cannot be $\left|C^{e}\right|$-projective, and we are done.

REMARK. In (5.10), "commutative" can be read in either the graded or ungraded sense. For $C$ is either concentrated in degree zero, or else it has characteristic two; in either case, commutativity of $C$ is synonymous with commutativity of $|C|$.

(5.11) TheOREM. Let $A$ be a $K$-algebra, finitely generated as a $K$-module, where $K$ is simple. The following are equivalent:

(1) $A$ is $K$-separable.

(2) $A$ is a finite product of simple $K$-algebras $A_{i}$; for each $i$, Center $A_{i}$ is a separable field extension of $K_{0}$, and CENTER $A_{i}=K \otimes_{K_{0}}\left(\right.$ Center $\left.A_{i}\right)$.

(3) For any algebraically closed field $F \supset K_{0},(F) \otimes_{K_{0}} A$ is a finite product of algebras of the types:

(a) $\operatorname{END}_{F}(W)$,

(b) $\mathrm{END}_{F}(V) \otimes_{F} F\langle 1\rangle$,

where $W$ and $V=\left(V_{0}\right)$ are finite-dimensional F-vector spaces. If $K=\left(K_{0}\right)$ and Char. $K \neq 2$, both types can occur, but if $K_{1} \neq 0$ only type (b) occurs, and if $K=\left(K_{0}\right)$ and Char. $K=2$, only type (a) occurs.

Proof. (1) $\Rightarrow(2)$. Assuming (1), we saw in (5.7(ii)) that every $A^{e}$-module is projective. From this it follows by a standard argument that the subgroup $\mathfrak{A}$ of $A$ generated by all simple $A^{e}$-submodules is a direct sum of simple submodules, and that $\mathfrak{A}=A$. Thus $A$ is a direct sum of simple $A^{e}$-submodules, that is, simple twosided ideals $A_{i}$. Each $A_{i}$ is a quotient $A / \coprod_{j \neq i} A_{j}$ of $A$, so that each $A_{i}$ inherits the structure of simple $K$-algebra, and the projections induce an isomorphism $A \rightarrow \prod A_{i}$.

For the rest, let us simplify the notation by letting $A$ denote any $A_{i}$. Then $C=A^{A}$ is simple, so that $A$ is $C$-projective, and therefore $C$ is $K$-separable by (4.17). If $F$ is an algebraic closure of $K_{0},(F) \otimes_{K_{0}} C$ is $(F) \otimes_{K_{0}} K$-separable, and $(F) \otimes_{K_{0}} K$ is simple by (5.9). The part of (1) $\Rightarrow(2)$ already proved shows that $(F) \otimes_{K_{0}} C$ is a product of simple $(F) \otimes_{K_{0}} K$-algebras, and therefore its degree zero term, $F \otimes_{K_{0}} C_{0}$, is a product of copies of $F$. Thus $F \otimes_{K_{0}} C_{0}$ is $F$-separable, and consequently $C_{0}$ is $K_{0}$-separable. That $C_{0}$ is a separable $K_{0}$-algebra if and only if $C_{0} / K_{0}$ is a separable field extension is proved in [3, III.3.3].

(2) $\Rightarrow$ (3). We may assume $A$ is simple, with $C_{0} / K_{0}$ a separable field extension and $A^{A}=C=\left(C_{0}\right) \otimes_{K_{0}} K$. If $F \supset K_{0}$ is algebraically closed, $\left[F \otimes_{K_{0}} C_{0}: F\right]=\left[C_{0}: K_{0}\right]$ $=\left[C_{0}: K_{0}\right]_{s}$, and $F \otimes_{K_{0}} C_{0}=\prod F_{\sigma}$, where the $F_{\sigma}$ are copies of $F$ indexed by imbeddings $\sigma: C_{0} \rightarrow F$ over $K_{0}$. Thus $(F) \otimes_{K_{0}} C=\prod F_{\sigma} \otimes_{K_{0}} K$ and $(F) \otimes_{K_{0}} A$ 
$=\prod F_{\sigma} \otimes_{C_{0}} A$. Now $A_{\sigma}=F_{\sigma} \otimes_{C_{0}} A$ is simple by (5.9), and its CENTER is $F \otimes_{C_{0}} C=F \otimes_{K_{0}} K$. Theorem (5.5) shows that $A_{\sigma}$ is one of the given types.

(3) $\Rightarrow(1)$ is clear.

(5.12) COROLlary. Let $A$ be a $K$-algebra and assume either

(i) $|K|$ is commutative and $A$ is finitely $K$-presented, or

(ii) $K$ is Noetherian and $A$ is a finitely generated $K$-module.

Then the following are equivalent:

(1) $A$ is $K$-separable.

(2) For every maximal ideal $m$ of $K, A / m A$ is a finite product of simple graded $K / m$-algebras, each with Center a separable field extension of $K_{0} / m_{0}$ and CENTER $=K / m \otimes_{K_{0} / m_{0}}$ (Center).

(5.13) Corollary. Let $A$ be a separable $K$-algebra, finitely presented as $K$ module, where $K=\left(K_{0}\right)$. Then $|A|, A_{0},\left|A^{A}\right|$ and $\left|A^{A_{0}}\right|$ are $|K|$-separable, and $A^{A}$ and $A^{A_{0}}$ are $K$-separable.

6. Azumaya algebras and the Brauer-Wall group. Throughout this section $k$ denotes a commutative ring, concentrated in degree zero. We will call a $k$-algebra $A$ CENTRAL if $k \rightarrow$ CENTER $A$ is an isomorphism.

(6.1) Theorem And Definition. $A$ is an Azumaya $k$-algebra if it satisfies the following conditions, which are equivalent:

(1) $A$ is CENTRAL and $k$-separable and finitely generated as a $k$-module.

(2) $A$ is CENTRAL, and a GENERATOR in MOD- $A^{e}$.

(3) $A$ is a FAITHFULLY projective $k$-module and the natural representation $A^{e} \rightarrow \operatorname{END}_{k}(A)$ is an isomorphism.

(4) $A$ is an invertible $A^{e}-k$-bimodule and

$$
A^{e} \text {-Mod } \underset{A \otimes_{k}}{\stackrel{M \mapsto M^{A}}{\rightleftarrows}} k \text {-Mod }
$$

are $k$-equivalences.

(5) $A$ is a finitely generated projective $k$-module, and for every maximal ideal $m$ of $k, A / m A$ is a CENTRAL simple $k / m$-algebra. (Recall that simple means no proper graded ideals.)

(6) There is a $k$-algebra $B$ and a FAITHFULL $Y$ projective $k$-module $P$ such that $A \otimes_{k} B \approx \mathrm{END}_{k}(P)$.

The proof of equivalence has essentially two ingredients: the Morita theory of $\S 3$, and the structural theorems, (5.11) and (5.12). We omit the details, but point out the following lemma, useful also in other contexts.

(6.2) LEMMA. With the hypothesis of (6.1(1)), every maximal two-sided ideal of $A^{e}$ is of the form $m A^{e}$ for some maximal ideal $m$ of $k$. 
Of course this follows from the Morita equivalence of (6.1(4)), but to prove it from the hypotheses of (6.1(1)), which are the usual definition of "Azumaya", we need the structure theory of $\S 5$. Indeed, let $\mathscr{M}$ be a maximal two-sided ideal of $A^{e}$, and put $m=\mathscr{M} \cap k$. The lemmas of $\S 3$ show that $m$ is a maximal ideal of $k$, and the structure theory of $\S 5$ shows that $A^{e} / m A^{e}$ is simple; (6.2) follows.

The Morita equivalence of (6.1(4)) has several further consequences.

(6.3) Corollary. Let $A$ and $B$ be k-algebras with A Azumaya. Then

(i) $\mathfrak{A} \mapsto A \mathfrak{A}$ is a bijection, \{ideals of $k\} \rightarrow\{$ two-sided ideals of $A\}$.

(ii) $\mathfrak{B} \mapsto A \otimes \mathfrak{B}$ is a bijection, \{two-sided ideals of $B\} \rightarrow\{$ two-sided ideals of $\left.A \otimes_{k} B\right\}$.

(6.4) Corollary. If $A \subset B$ are $k$-algebras with $A$ Azumaya, $A \otimes_{k} B^{A} \approx B$.

(6.5) Corollary. Every endomorphism of an Azumaya k-algebra is an automorphism.

Proof. If $f$ is a $k$-algebra endomorphism of $A, \operatorname{ker} f$ is a two-sided ideal, hence ker $f=0$ by (6.3(i)). Then $A \approx A^{f A} \otimes_{k} f A$ by (6.4). Since $A$ and $f A$ are $k$-modules of the same rank, $A^{f A}$ is a rank one $k$-module. With $k \subset A^{A} \subset A^{f A}$, this implies $A^{f A} \approx k$, and we are done.

Let $A$ be a $k$-algebra. The next few results concern $A_{\text {ut }}$-alg $(A)$, particularly when $A$ is Azumaya. For $u \in h U(A)$, the group of homogeneous units of $A$, we define the inner automorphism $\alpha_{u}$ by $\alpha_{u} a=-1^{u a} u a u^{-1}(a \in h A)$. Then $u \mapsto \alpha_{u}$ is a homomorphism $i: h U(A) \rightarrow \operatorname{Aut}_{k \text {-alg }}(A)$. Denote the image of $i$ by $\operatorname{In} \operatorname{Aut}_{k \text {-alg }}(A)$. The kernel of $i$ is $\left\{u \in h U(A) \mid a=-1^{u a} u a u^{-1} \forall a \in h A\right\}=h U\left(A^{A}\right)$, hence the sequences

$$
\begin{gathered}
1 \longrightarrow h U\left(A^{A}\right) \longrightarrow h U(A) \stackrel{i}{\longrightarrow} \operatorname{Aut}_{k \text {-alg }}(A), \\
1 \longrightarrow U\left(\left[A^{A}\right]_{0}\right) \longrightarrow U\left(A_{0}\right) \stackrel{i_{0}}{\longrightarrow} \operatorname{Aut}_{k \text {-alg }}(A)
\end{gathered}
$$

are exact, where $i_{0}$ is simply the restriction of $i$. Let In $\operatorname{Aut}_{k \text {-alg }}^{0}(A)$ denote the image of $i_{0}$, and, similarly, let In $\operatorname{Aut}_{k \text {-alg }}^{1}(A)=\left\{\alpha_{u} \mid u \in A_{1}\right\}$.

Let $\operatorname{Pic}_{k}(A)$ denote the set of isomorphism classes $[P]$ of invertible $A_{k} A$ bimodules $P$ (Definition 3.19). The condition for $[P]=[Q]$ in $\operatorname{Pic}_{k}(A)$ is $P \approx Q$ in $A^{e}$-Mod. $\operatorname{Pic}_{k}(A)$ is a group with multiplication induced by $\otimes_{A}$ and identity $[A]$, and $[P]^{-1}=\left[\operatorname{HOM}_{A}(P, A)\right]=\left[\operatorname{HOM}_{A}(P, A)\right]$. Pic $c_{k}(A)$ is isomorphic to the group of isomorphism classes of $\tau$ - $k$-autoequivalences of Mod- $A$ under composition.

As an example, consider $\operatorname{Pic}(k)=\operatorname{Pic}_{k}(A)$. If $[P] \in \mathrm{Pic}(k), P$ is a rank one projective. If $k$ is connected $\left({ }^{7}\right)$, then $P$ is concentrated in degree zero or one; $|P|$ is an invertible $|k|$-module, and $[P]_{\text {Pic }(k)} \mapsto\left([P]_{\text {Pic }|k|}, i\right)$, where $P_{i+1}=0$, defines an isomorphism, Pic $(k) \rightarrow \operatorname{Pic}|k| \oplus Z / 2 Z$. Clearly, we can never have Pic $(k)=\{0\}$, since $\tau k$ represents a nontrivial element.

$\left({ }^{7}\right) k$ is said to be connected if Spec $|k|$ is a connected topological space. This is equivalent to the requirement that $e^{2}=e, e \in k$, implies $e=0$ or $e=1$. Local rings and integral domains are connected. 
Let $\mathrm{PIC}_{k}(A)$ denote the quotient of $\mathrm{Pic}_{k}(A)$ obtained by the equivalence relation of isomorphism in $A^{e}$-MOD. It is convenient to introduce also an intermediate group. Let $A-\mathscr{M O O D}$ be the category with the same objects as $A-\mathrm{MOD}$, but with homogeneous morphisms only, and let $\mathscr{P} \mathscr{S} \mathscr{C}_{k}(A)$ denote the quotient of $\operatorname{Pic}_{k}(A)$ by the equivalence relation of isomorphism in $A^{e}-\mathscr{M O D}$.

For example, PIC $(k)=$ Pic $|k|$. Also, since $\mathscr{P} \mathscr{I} \mathscr{C}(k)$ is caught between Pic $(k)$ and $\mathrm{PIC}(k), \mathscr{P} \mathscr{S} \mathscr{C}(k)=\mathrm{PIC}(k)$ when $k$ is connected. In general, $\mathscr{P} \mathscr{I} \mathscr{C}(A)$ is obtained simply by introducing the relations $[P]=[\tau P]$ in Pic $(A)$.

If $P$ is an invertible $A_{k} A$-bimodule and $\alpha, \beta \in$ Aut $_{k \text {-alg }}(A)$ we write ${ }_{\alpha} P_{\beta}$ for the $A_{k} A$-bimodule whose additive group is $P$, with operations $a \cdot x=(\alpha a) x$ and $x \cdot a$ $=x(\beta a)(a \in A, x \in P)$. As in the ungraded case (cf. [3, II.7.2]) we have

(6.7) LemMA. If $\alpha, \beta, \gamma \in \mathrm{Aut}_{k \text {-alg }}(A)$ then

(i) ${ }_{\alpha} A_{\beta} \approx_{\gamma \alpha} A_{\gamma \beta}$ (degree zero bimodule isomorphism).

(ii) ${ }_{1} A_{\alpha} \otimes_{A 1} A_{\beta} \approx{ }_{1} A_{\alpha \beta}$ (degree zero bimodule isomorphism).

(iii) There is a homogeneous isomorphism ${ }_{1} A_{\alpha} \rightarrow{ }_{1} A_{1}$ of degree $j \Leftrightarrow$

$$
\alpha \in \operatorname{In} \operatorname{Aut}_{k-\text { alg }}^{j}(A) .
$$

(iv) If $P$ and $Q$ are invertible bimodules and $P \approx Q$ as left $A$-modules, there exists $\alpha \in \mathrm{Aut}_{k-\mathrm{alg}}(A)$ such that $P \approx_{1} Q_{\alpha}$ as bimodules. If the left $A$-isomorphism is homogeneous the bimodule isomorphism is homogeneous of the same degree.

(6.8) CoROllary. $\alpha \mapsto\left[{ }_{1} A_{\alpha}\right]$ defines homomorphisms $\varphi$ and $\varphi^{0}$ making

$$
1 \longrightarrow \operatorname{In} \operatorname{Aut}_{k-\text {-alg }}^{0}(A) \longrightarrow \operatorname{Aut}_{k-\Omega l g}(A) \stackrel{\varphi^{0}}{\longrightarrow} \operatorname{Pic}_{k}(A)
$$

and

$$
1 \longrightarrow \operatorname{In~Aut~}_{k-\mathrm{alg}}(A) \longrightarrow \operatorname{Aut}_{k-\mathrm{alg}}(A) \stackrel{\varphi}{\longrightarrow} \mathscr{P} \mathscr{I} \mathscr{C}_{k}(A)
$$

exact, and

$$
\operatorname{Im} \varphi^{0}=\{[P] \mid P \approx A \text { in } A-\operatorname{Mod}\}
$$

and

$$
\operatorname{Im} \varphi=\{[P] \mid P \approx A \text { in } A-\mathscr{M O D}\} .
$$

(6.9) COROLlary. If $A$ is an Azumaya $k$-algebra we have exact sequences

$$
1 \longrightarrow U(k) \longrightarrow U\left(A_{0}\right) \longrightarrow \operatorname{Aut}_{k-\text { alg }}(A) \stackrel{\psi^{0}}{\longrightarrow} \operatorname{Pic}(k)
$$

and

and

$$
1 \longrightarrow U(k) \longrightarrow h U(A) \longrightarrow \operatorname{Aut}_{k-\text { alg }}(A) \stackrel{\psi}{\longrightarrow} \mathscr{P} \mathscr{I} \mathscr{C}(k),
$$

and

$$
\operatorname{Im} \psi^{0}=\left\{[P] \mid A \otimes_{k} P \approx A \text { in } A \text {-Mod }\right\}
$$

$$
\operatorname{Im} \psi=\left\{[P] \mid A \otimes_{k} P \approx A \text { in } A-\mathscr{M O D}\right\} .
$$

Here $\psi$ and $\psi^{0}$ are defined by $\alpha \mapsto\left[{ }_{1} A_{\alpha}^{A}\right]$. 
Proof. This is immediate from (6.6) and (6.8), with the observation that when $A$ is Azumaya, $A \otimes_{k}$ induces an isomorphism $\operatorname{Pic}(k) \rightarrow \operatorname{Pic}_{k}(A)$ (hence also an isomorphism $\left.\mathscr{P} \mathscr{I} \mathscr{C}(k) \rightarrow \mathscr{P} \mathscr{I} \mathscr{C}_{k}(A)\right)$. The latter follows from the facts that $A \otimes_{k}$ is an equivalence and $\left(A \otimes_{k} M\right) \otimes_{A}\left(A \otimes_{k} N\right) \approx A \otimes_{k}\left(M \otimes_{k} N\right)$.

To illustrate (6.9), suppose there is a unit $u$ in $A_{1}$. Then $\psi^{0}\left(\alpha_{u}\right)=[\tau k]$. The fact that [ $\tau k]$ is in the image of $\psi^{0}$ says that $A \otimes_{k} \tau k \approx \tau A \approx A$ in $A$-Mod, and this is equivalent to the original hypothesis, that there be a unit in $A_{1}$. On the other hand, $\alpha_{u}$ is in the kernel of $\psi$. Hopefully this clarifies the relation between Pic $(k)$ and $U\left(A_{0}\right)$, and between $\mathscr{P} \mathscr{I} \mathscr{C}(k)$ and $h U(A)$, displayed in (6.9).

(6.10) Corollary ("Skolem-Noether"). If $A$ is an Azumaya k-algebra and $\mathscr{P} \mathscr{I} \mathscr{C}(k)=\{0\}$, the only endomorphisms of $A$ (as a graded $k$-algebra) are the inner automorphisms $\alpha_{u}$ by homogeneous units $u$.

We have seen that $\mathscr{P} \mathscr{I} \mathscr{C}(k)=$ Pic $|k|$ when $k$ is connected. In general, $\mathscr{P} \mathscr{I} \mathscr{C}(k)$ $=\{0\}$ is a stronger requirement than Pic $|k|=\{0\}$.

We now return to (6.1), and construct a group out of our Azumaya algebras.

(6.11) Proposition. Let $A$ and $B$ be Azumaya k-algebras, and write $\otimes$ for $\otimes_{k}$ and $\mathrm{END}$ for $\mathrm{END}_{k}$. The following are equivalent:

(i) $A \otimes B^{*} \approx \operatorname{END}(P)$ for some FAITHFULL $Y$ projective $k$-module $P$.

(ii) $A \otimes \operatorname{END}(P) \approx B \otimes \operatorname{END}(Q)$ for some FAITHFULLY projective $k$ modules $P$ and $Q$.

(iii) Mod- $A$ and Mod- $B$ are $\tau$-k-equivalent categories.

(iv) $A \approx \mathrm{END}_{B}(P)$ for some FAITHFULL Y projective right B-module $P$.

The proof of equivalence is straightforward from (6.1) and the Morita theory of $\S 3$. Write $A \sim B$ when the conditions listed in (6.11) are fulfilled. It is clear from (6.11) that $\sim$ is an equivalence relation, and that $\otimes_{k}$ induces a well-defined structure of an abelian group on the set of equivalence classes. This is the Brauer-Wall group, BW $(k)$, of $k$. If $A$ is an Azumaya $k$-algebra, write [ $A]$ for the corresponding element of BW $(k)$. Then, for example, $[A]=1 \Leftrightarrow A \sim k \Leftrightarrow A \approx \operatorname{END}_{k}(P)$ for some FAITHFULLY projective $P$, and $[B]^{-1}=\left[B^{*}\right]$.

If $K$ is a commutative $k$-algebra concentrated in degree zero, $K \otimes_{k}$ induces a homomorphism BW $(k) \rightarrow \mathrm{BW}(K)$, and this makes BW a functor, from commutative (ungraded) rings to abelian (ungraded) groups. Another such functor is $\mathrm{Br}$, where $\mathrm{Br}(k)$ denotes the Brauer group of $k$ (see [1], and Chapter III of [3]). All of our definitions have been made in such a way that they reduce, for objects concentrated in degree zero, to the corresponding ungraded notions: our construction of $\mathrm{BW}(k)$ "contains" the construction of $\mathrm{Br}(k)$. This means that concentrating an Azumaya $|k|$-algebra in degree zero yields an Azumaya $k$-algebra, and this induces an injective homomorphism $\mathrm{Br}(k) \rightarrow \mathrm{BW}(k)$. (This is, moreover, natural in $k$.) $\S 7$ is devoted to a description of the cokernel. 
7. Graded quadratic extensions and the exact sequence $0 \rightarrow \mathrm{Br} \rightarrow \mathrm{BW} \rightarrow Q_{2} \rightarrow 0$. Throughout, $k$ is commutative and concentrated in degree zero.

In this section we require basic facts about Galois extensions of rings, for which we refer chiefly to [4].

Suppose $k$ is not connected, and let $e^{\prime} \in k$ be a nontrivial idempotent. Then, setting $e^{\prime \prime}=1-e^{\prime}, k^{\prime}=k e^{\prime}$, and $k^{\prime \prime}=k e^{\prime \prime}$, we have $k \approx k^{\prime} \times k^{\prime \prime}$, a direct product of rings. Clearly $M \mapsto\left(e^{\prime} M, e^{\prime \prime} M\right)=\left(M / e^{\prime \prime} M, M / e^{\prime} M\right)$ is a category isomorphism $|k|$-Mod $\rightarrow\left|k^{\prime}\right|-\operatorname{Mod} \times\left|k^{\prime \prime}\right|-\operatorname{Mod}$; an inverse is given by $(R, S) \mapsto R \times S$. Equivalently this provides an isomorphism $k$-MOD $\rightarrow k^{\prime}$-MOD $\times k^{\prime \prime}$-MOD (where a morphism $(g, h)$ in $k^{\prime}$-MOD $\times k^{\prime \prime}$-MOD is homogeneous of degree $j$ if and only if both $g$ and $h$ are). In particular we get isomorphisms $k$-Mod $\rightarrow k^{\prime}$-Mod $\times k^{\prime \prime}$-Mod, and $k$-Alg $\rightarrow k^{\prime}$-Alg $\times k^{\prime \prime}$-Alg. All of these will be denoted $X \mapsto\left(X^{\prime}, X^{\prime \prime}\right)$.

For a finitely generated projective $k$-module $M$ we let $[M: k]$ denote the rank of $M$; this is a continuous (i.e. locally constant) function, Spec $|k| \rightarrow Z$. In the situation $k=k^{\prime} \times k^{\prime \prime}$ above, $M$ is finitely generated and projective $\Leftrightarrow M^{\prime}$ and $M^{\prime \prime}$ are so (over $k^{\prime}$ and $k^{\prime \prime}$ respectively). Moreover, a prime $\mathfrak{p}$ of $k$ corresponds either to a prime $\mathfrak{g}^{\prime}$ of $k^{\prime}$ or to a prime $\mathfrak{p}^{\prime \prime}$ of $k^{\prime \prime}$ (the decomposition $k=k^{\prime} \times k^{\prime \prime}$ breaks Spec $|k|$ into a disjoint union of open-and-closed spaces which can be identified with Spec $\left|k^{\prime}\right|$ and Spec $\left.\left|k^{\prime \prime}\right|\right)$ and $[M: k] \mathfrak{p}$ is $\left[M^{\prime}: k^{\prime}\right] \mathfrak{p}^{\prime}$ or $\left[M^{\prime \prime}: k^{\prime \prime}\right] \mathfrak{p}^{\prime \prime}$ as the case may be. In particular, $[M: k]$ is a constant, $n$, if and only if $\left[M^{\prime}: k^{\prime}\right]=n=\left[M^{\prime \prime}: k^{\prime \prime}\right]$.

(7.1) LemMA. Let $L$ be a $k$-algebra, projective of rank 2 as a $k$-module. Then $|L|$ is commutative, and $L$ is a $k$-separable $\Leftrightarrow|L|$ is $|k|$-separable.

Proof. Localize.

We call a $k$-algebra $L$ a quadratic extension (abbreviated QE) of $k$ if it is separable, and projective of rank 2 as a $k$-module. QE's are FAITHFULLY projective (e.g., by [2, III.7.2]), and if $k=k^{\prime} \times k^{\prime \prime}, L$ is a QE of $k \Leftrightarrow L^{\prime}$ is a QE of $k^{\prime}$ and $L^{\prime \prime}$ is a QE of $k^{\prime \prime}$.

(7.2) Lemma. If $k$ admits a $Q E, L$, with $L_{1}$ faithful, then $2 \in U(k)$. In particular, all $Q E$ 's are concentrated in degree zero in either of the following situations:

(1) $k$ is connected and $2 \notin U(k)$.

(2) $2 \in \operatorname{rad}(k)=$ intersection of all maximal ideals of $k$.

Proof. If $L_{1}$ is faithful, $L / m L$ has nonzero degree one part for every maximal ideal $m$ of $k$. If $2 \notin U(k), 2$ is in some $m$, and we contradict (5.10).

The key fact is

(7.3) Proposition. If $L$ is a $Q E$ of $k$, there is a unique $k$-algebra automorphism $\sigma=\sigma(L)$ of $L$ of order two such that $L^{\sigma}=\{x \in L \mid \sigma x=x\}=k$.

Proof. Since $k$ is a direct summand of $L_{0}$ and $[L: k]=2,\left[L_{1}: k\right]$ can take no values except 0 and 1 . If $k$ is connected, [ $\left.L_{1}: k\right]$ is constant, and if not we can write $k=k^{\prime} \times k^{\prime \prime}$ so that $\left[L_{1}^{\prime}: k^{\prime}\right]=1$ and $L_{1}^{\prime \prime}=0$. Clearly in the latter case the existence and 
uniqueness of $\sigma(L)$ are equivalent to the existence and uniqueness of $\sigma\left(L^{\prime}\right)$ and $\sigma\left(L^{\prime \prime}\right)$. Thus whether or not $k$ is connected it suffices to treat two cases: $\left[L_{1}: k\right]=1$, and $L_{1}=0$.

If $\sigma$ is as in the statement and $x \in L$ then $\sigma x+x \in L^{\sigma}=k$. In particular, $\sigma x+x \in L_{1} \cap k=\{0\}$ for all $x \in L_{1}$, i.e. $\sigma \mid L_{1}=-1$. Now if $\left[L_{1}: k\right]=1$ it follows that $L_{0}=k$, and we have established uniqueness of $\sigma=1_{L_{0}} \oplus-1_{L_{1}}$ in this case. It remains to see that $L^{\sigma}=k$. This is clear unless $\exists 0 \neq x \in L_{1}$ with $x=-x$. But when $\left[L_{1}: k\right]$ $=1,2 \in U(k)$ by (7.2), and this rules out 2-torsion.

Thus, for the rest of the proof, $L=\left(L_{0}\right)$. Assume first that $L$ is connected. By a theorem of Janusz [5, Theorem 1] there is a connected Galois extension $E$ of $k$, with group $G$, say, such that $k \subset L \subset E$. The subgroup $H=\{\sigma \in G \mid \sigma x=x \forall x \in L\}$ of $G$ has index $[L: k]=2$ and is therefore normal. Consequently $L=E^{H}$ is a Galois extension of $k$ with group $G / H$ of order two. The generator of this group is a candidate for $\sigma(L)$, and it is the only candidate by [4, Corollary 3.3].

If $k$ is still connected but $L$ is not, it is easy to see that $L=k \times k$, and $\sigma(L)$ is the transposition $(x, y) \mapsto(y, x)$.

This completes the proof for connected $k$. Note that this furnishes uniqueness for all $k$, for to prove uniqueness we can localize, and local rings are connected. Also the connected case extends immediately to the case where $k$ is Noetherian, for $k$ is then a finite product of connected rings. Thus to complete the proof in the general case it suffices to show the following: if $L$ is a QE of $k$ there is a Noetherian subring $k^{\prime}$ of $k$ and a $\mathrm{QE} L^{\prime}$ of $k^{\prime}$ contained in $L$ such that the induced map $k \otimes_{k^{\prime}} L^{\prime} \rightarrow L$ is an isomorphism. (For then the above arguments furnish $\sigma\left(L^{\prime}\right)$, and we can take $\sigma(L)=k \otimes_{k^{\prime}} \sigma\left(L^{\prime}\right)$.) This is a more or less standard descent argument, which we omit here.

(7.4) Corollary. A $k$-algebra $L$ is a $Q E$ of $k \Leftrightarrow|L|$ is a Galois extension of $|k|$ with group of order two.

(7.3) and (7.4) give us a group of order 2 (generated by $\sigma(L)$ ) whenever we have a QE, $L$. Choose once and for all a fixed group $\pi$ of order 2 with which we can identify all such groups.

Let $L^{1}$ and $L^{2}$ be QE's of $k$ and define

$$
L^{1}|*| L^{2}=\left[\left|L^{1}\right| \otimes_{k}\left|L^{2}\right|\right]^{\sigma_{1} \otimes \sigma_{2}}
$$

where $\sigma_{i}=\sigma\left(L^{i}\right)$. Although we use the ungraded $\otimes_{k}$ to form $L^{1}|*| L^{2}$, we interpret $L^{1}|*| L^{2}$ as a graded $k$-algebra, i.e., with the grading inherited from that of $L^{1}$ and $L^{2}$.

(7.5) THEOREM. If $L^{1}$ and $L^{2}$ are $Q E$ 's of $k$, so is $L^{1}|*| L^{2}$. $|*|$ induces on the set of $k$-algebra-isomorphism classes of $Q E ' s$ the structure of an abelian group, $Q_{2}(k)$, of exponent two.

Proof. $\left|L^{1}\right| \otimes\left|L^{2}\right|$ is a Galois extension of $k$ with group $\pi \times \pi$, and consequently, $L^{1}|*| L^{2}$ is a Galois extension: $\sigma_{1} \otimes 1$ and $1 \otimes \sigma_{2}$ have the same restriction 
to $L^{1}|*| L^{2}$, and this automorphism is the generator of the Galois group. (See [1, Theorem A8].) To complete the proof we show

(a) $L|*|(k \times k) \approx L$ for any QE, $L$,

(b) $L|*| L \approx(k \times k)$ for any QE, $L$, and

(c) $|*|$ is associative (up to $k$-isomorphism).

Proof of (a). Clearly $|L| \otimes|k \times k| \approx|L| \times|L|$, and the automorphism of $|L| \times|L|$ which corresponds to $\sigma(L) \otimes \sigma(k \times k)$ is $(x, y) \mapsto(\sigma y, \sigma x)$. Thus $L|*|(k \times k) \approx\{(x, \sigma x) \in L \times L\} \approx L$.

Proof of (b). By criterion (e) of [4, Theorem 1.3], $x \otimes y \mapsto(x y, x(\sigma y))$ defines a $k$-isomorphism $|L| \otimes|L| \rightarrow|L \times L|$. Clearly the subset of $|L \times L|$ corresponding to $L|*| L$ is precisely $\left|L^{\sigma} \times L^{\sigma}\right|=(k \times k)$.

Proof of (c). One sees easily that $\left[L^{1}|*| L^{2}\right]|*| L^{3}$ and $L^{1}|*|\left[L^{2}|*| L^{3}\right]$ are both $k$-isomorphic to $\left[\left|L^{1}\right| \otimes\left|L^{2}\right| \otimes\left|L^{3}\right|\right]^{K}$ where $K$ is the kernel of the map $(x, y, z) \rightarrow x y z, \pi \times \pi \times \pi \rightarrow \pi$.

Unfortunately, $\boldsymbol{Q}_{2}$ is not quite the group we need to describe the cokernel of $\mathrm{Br} \rightarrow \mathrm{BW}$. We define a new product, for QE's $L^{1}$ and $L^{2}$ of $k$, as follows:

$$
L^{1} * L^{2}=\left[L^{1} \otimes L^{2}\right]^{\sigma_{1} \otimes \sigma_{2}}
$$

where $\sigma_{i}=\sigma\left(L^{i}\right)$. The distinction is that here we take the tensor product as graded algebras.

(7.6) Lemma. Let $G$ be an abelian group of exponent 2 , let $\mu$ be a map $G \times G \rightarrow G$, let $\partial$ be a homomorphism $G \rightarrow Z / 2 Z$, and assume there is an element $\varepsilon \in \operatorname{ker} \partial$ such that $\mu(x, y)=x+y+\partial x \cdot \partial y \cdot \varepsilon \forall x, y \in G$. Then $G$, with + replaced by $\mu$, is an abelian group. If $1 \notin \operatorname{Im} \partial$, or if $\varepsilon=0$, then $(G,+)$ and $(G, \mu)$ coincide; conversely, if $\exists z$ with $\partial z=1$, then $\mu(z, z)=\varepsilon$, so that if also $\varepsilon \neq 0,(G, \mu)$ has exponent 4 .

The proof is a triviality; the inverse of $x$ in the new group is $x+\partial x \cdot \varepsilon$. Since this lemma is unlikely ever to find application elsewhere, we hasten to describe how it applies to our present situation. Assume, for simplicity, that $k$ is connected. Now * and $|*|$ differ only if $k$ has a QE with nonzero degree one term, so we may as well assume also that $2 \in U(k)$, by (7.2). Then $F=|k\langle-1\rangle|$ is a QE of $k$. If $L^{1}$ and $L^{2}$ are QE's of $k, L^{1} * L^{2}=L^{1}|*| L^{2}$ unless $L_{1}^{1} \neq 0 \neq L_{1}^{2}$. In the latter case it is not hard to see that $L^{1} * L^{2}=L^{1}|*| L^{2}|*| F$. Thus, in any case, $L^{1} * L^{2}$ is a $\mathrm{QE}$ of $k$, so that * defines a map $G \times G \rightarrow G$, where $G=Q_{2}(k)$. The map which assigns to each QE the rank of its degree one term is a homomorphism $\partial: G \rightarrow Z / 2 Z$, and $F$ plays the role of $\varepsilon$. With this dictionary, (7.5) and (7.6) imply the following theorem in the case where $k$ is connected.

(7.7) THEOREM. If $L^{1}$ and $L^{2}$ are $Q E$ 's of $k$, so is $L^{1} * L^{2} . *$ induces on the set of $k$-algebra-isomorphism classes of $Q E$ 's the structure of an abelian group, $Q_{2}(k)$, of exponent $\leqq 4$.

The general case follows immediately, since for each statement to be proved we can break $k$ up into pieces over which the intervening QE's have degree one parts of constant rank. 
Let $E$ denote $\operatorname{END}_{k}(V)$ where $V_{0}=k=V_{1}$. For any $k$-algebra $A$ define $L(A)=A^{A_{0}}$ and $L^{\prime}(A)=L\left(A \otimes_{k} E\right)$. When $A$ is Azumaya, $L$ and $L^{\prime}$ "commute" (in the obvious sense) with localizations at primes of $k$. We will call a finitely generated projective $k$-module $P$ nondegenerate if $\left[P_{0}: k\right]$ and $\left[P_{1}: k\right]$ are both strictly positive. (Equivalently, both $P_{0}$ and $P_{1}$ are FAITHFULLY projective; cf. (3.15(i)) and [2, III.7.2].)

(7.8) TheOrem. Let $A$ and $B$ be Azumaya $k$-algebras. Then

(i) $A$ is a nondegenerate $k$-module $\Leftrightarrow L(A)$ is a $Q E$ of $k$.

(ii) If $A$ and $B$ are nondegenerate, so is $A \otimes B$, and $L(A \otimes B)=L(A) * L(B)$.

(iii) Let $P$ be a finitely generated projective $k$-module. $P$ is nondegenerate $\Leftrightarrow A$ $=\mathrm{END}_{k}(P)$ is nondegenerate, in which case $L(A)=(k \times k)$.

Proof. (i) follows from (6.1(5)), (5.6) and (5.13).

(ii) The first statement is trivial. The second reduces to the situation where $L(A)$ and $L(B)$ have degree one terms of constant rank. Note first that we have

( $\alpha) A^{L(A)}=A_{0}$, and

(B) $L(A \otimes B) \subset(A \otimes B)^{A_{0} \otimes B_{0}}=L(A) \otimes L(B)$.

(It suffices to check $(\alpha)$ modulo maximal ideals of $k$, since clearly $A_{0} \subset A^{L(A)}$, where it is true by (6.1(5)) and (5.5). ( $\beta)$ is clear since $A_{0} \otimes B_{0} \subset(A \otimes B)_{0}$ and $A_{0}$ and $B_{0}$ are $k$-separable by (5.13).)

The proof breaks into two cases. Assume first that $\left[L(A)_{1}: k\right]$ and $\left[L(B)_{1}: k\right]$ are not both 1 . Then

$$
|L(A \otimes B)| \subset|L(A) \otimes L(B)|=|L(A)| \otimes|L(B)| .
$$

The latter is a Galois extension of $|k|$ with group $\pi \times \pi$. Since $\pi \times \pi$ has three subgroups of order two, $|L(A)| \otimes|L(B)|$ has precisely three separable subalgebras of rank two $\left({ }^{8}\right)$. Since $|L(A \otimes B)|,|L(A)| \otimes 1,1 \otimes|L(B)|$ and $|L(A) * L(B)|$ are four such subalgebras, it suffices to show that $L(A \otimes B) \neq L(A) \otimes 1$ (for then by symmetry $L(A \otimes B) \neq 1 \otimes L(B)$, and clearly $L(A) \otimes 1 \neq L(A) * L(B) \neq 1 \otimes L(B))$. But if $L(A \otimes B)=L(A) \otimes 1$, we have

$$
(A \otimes B)_{0}=(A \otimes B)^{L(A \otimes B)}=(A \otimes B)^{L(A) \otimes 1}=A^{L(A)} \otimes B=A_{0} \otimes B .
$$

But this implies $A_{1} \otimes B_{1}=0$, contradicting nondegeneracy.

In the case where $\left[L(A)_{1}: k\right]=1=\left[L(B)_{1}: k\right]$, we have isomorphisms $A_{0} \otimes L(A) \rightarrow A$ and $B_{0} \otimes L(B) \rightarrow B$, for modulo maximal ideals of $k$ we are in Case (1) of Theorem (5.5). For the same reason, $\sigma(L(A))$ is 1 on $L(A)_{0}=k$ and -1 on $L(A)_{1}$ (and similarly for $B$ ). Thus

$$
L(A) * L(B)=[L(A) \otimes L(B)]_{0}=k \oplus\left[L(A)_{1} \otimes L(B)_{1}\right] .
$$

$\left({ }^{8}\right)$ See [4, $\$ 2$ and Lemma 4.1]. A bit of caution is required because of possible idempotents in the subalgebras in question; in the language of [4], we must be able to assume that these subalgebras are " $\pi \times \pi$-strong". The reduction to this case is immediate. 
It suffices to show that this is contained in $L(A \otimes B)$. Since

$$
\begin{aligned}
(A \otimes B)_{0} & =\left(A_{0} \otimes B_{0}\right) \oplus\left(A_{1} \otimes B_{1}\right) \\
& =\left(A_{0} \otimes B_{0}\right) \oplus\left(A_{0} \otimes L(A)_{1} \otimes B_{0} \otimes L(B)_{1}\right) \\
& =A_{0} \otimes B_{0} \otimes(L(A) * L(B)),
\end{aligned}
$$

it suffices to note that $L(A) * L(B)$ is contained in $(A \otimes B)^{A_{0} \otimes B_{0}}=L(A) \otimes L(B)$ and in $(A \otimes B)^{L(A) * L(B)}$, and these are both clear.

(iii) The first statement is clear. For the second, we can realize $A$ as a matrix algebra over $k$, with degree zero term

$$
\left(\begin{array}{cc}
\mathrm{END}_{k}\left(P_{0}\right) & 0 \\
0 & \operatorname{END}_{k}\left(P_{1}\right)
\end{array}\right) .
$$

By nondegeneracy, both of the blocks $\operatorname{END}_{k}\left(P_{i}\right)$ are nonzero, so that $A^{A_{0}}$ $=k \times k \subset A_{0}$.

(7.9) Corollary. (i) If $A$ is a nondegenerate Azumaya k-algebra, $L(A)=L^{\prime}(A)$.

(ii) For any Azumaya $k$-algebras $A$ and $B, L^{\prime}(A \otimes B)=L^{\prime}(A) * L^{\prime}(B)$.

(7.10) THEOREM. $L^{\prime}$ induces a homomorphism making $0 \rightarrow \mathrm{Br}(k) \rightarrow \mathrm{BW}(k)$ $\stackrel{L^{\prime}}{\rightarrow} Q_{2}(k) \rightarrow 0$ exact. The sequence is natural in $k$, and splits when $k$ has characteristic two.

Proof. There are essentially two things to show: surjectivity of $L^{\prime}$, and exactness at BW $(k)$. Clearly for surjectivity of $L^{\prime}$ it is enough to note that every QE with degree one term of constant rank is in the image. If $\left[L_{1}: k\right]=1, L$ is itself an Azumaya $k$-algebra, and $L^{\prime}(L)=L(L)=L$. If $L$ is a QE concentrated in degree zero, let $A$ denote the crossed product of $L$ with its Galois group: $A=L v_{1} \oplus L v_{\sigma}$ with $a v_{i} \cdot b v_{j}=a i(b) v_{i j}$ and the indicated grading: $\partial v_{i}=\delta_{\sigma, i}$. Then $A$ is a nondegenerate Azumaya $k$-algebra, and $L^{\prime}(A)=L(A)=L$.

REMARK. When $k$ has characteristic $2, k\{a\}$ is a QE of $k$ for any $a \in k$, and in fact all QE's are of this form; the crossed product described above is then

$$
\left[\frac{1, a}{k}\right]
$$

When $2 \in U(k),|k\langle a\rangle|$ is a $\mathrm{QE}$ for any $a \in U(k)$, and the crossed product is

$$
\left(\frac{1,-a}{k}\right)
$$

A similar description can be given for arbitrary $k$. We do not digress to include the details here.

Finally, we prove exactness of $\mathrm{Br}(k) \rightarrow \mathrm{BW}(k) \rightarrow Q_{2}(k)$. Clearly the composition is zero. Conversely, let $A$ be an Azumaya $k$-alegbra such that $L^{\prime}(A)$ 
$=k \times k \subset A_{0}$. We can assume $A$ is nondegenerate, since $[A]=[A \otimes E]$ in $\mathrm{BW}(k)$. Thus we have

$$
\text { Center } A_{0}=A^{A_{0}}=k e \oplus k(1-e) \subset A_{0}, \quad e \in A_{0}, e \notin k, e^{2}=e, 0 \neq e \neq 1 .
$$

We must produce an Azumaya $k$-algebra $B=\left(B_{0}\right)$ such that $[A]=[B]$ in $\mathrm{BW}(k)$.

Notice that $e$ remains nontrivial when we localize at primes of $k$ : nondegeneracy of $A$ implies that $L\left(A_{m}\right)=L(A)_{m}$ has rank two at every prime $m$. Now set $P=e A$ and $B=$ END $_{A}(P)$. Clearly $P$ is a FAITHFULLY projective right $A$-module, and we are done by criterion (4) of (6.11) once we show that $B_{1}=0$. Since $B=\mathbf{E N D}_{A}(e A)$ $=e A e$, it remains to show that $e A_{1} e=0$. It suffices to see this modulo maximal ideals of $k$, and we can even assume $k$ is an algebraically closed field. Then by (5.11(3)) there are two cases to consider: $A=\operatorname{END}_{k}(W)$ and $A=\left(\operatorname{End}_{k}(V)\right) \otimes k\langle 1\rangle$. The second case is immediately ruled out: the center of the degree zero term of $\left(\operatorname{End}_{k}(V)\right) \otimes k\langle 1\rangle$, namely $k$, has no nontrivial idempotents. (This amounts to the remark that $k\langle 1\rangle$ is not in the kernel of $L^{\prime}$.) In the first case we can represent $A$ as a matrix algebra $\mathbf{M}_{p+q}(k)$ with degree zero term

$$
\left(\begin{array}{cc}
\mathbf{M}_{p}(k) & 0 \\
0 & \mathbf{M}_{q}(k)
\end{array}\right)
$$

When $p \neq 0 \neq q$ there are two idempotents: $e^{\prime}=\left(\begin{array}{cc}I_{p} & 0 \\ 0 & 0\end{array}\right)$ and $e^{\prime \prime}=\left(\begin{array}{ll}0 & 0 \\ 0 & I_{q}\end{array}\right)$, and clearly $e^{\prime} A e^{\prime}$ and $e^{\prime \prime} A e^{\prime \prime}$ are contained in $A_{0}$. This completes the proof.

To compute $\mathrm{BW}(k)$ by means of the exact sequence $0 \rightarrow \mathrm{Br} \rightarrow \mathrm{BW} \rightarrow Q_{2} \rightarrow 0$, we need additional information about $Q_{2}$. Actually, as indicated in the Introduction, $Q_{2}(k)$ can be computed quite explicitly in terms of certain standard arithmetic invariants of $k$. This will be presented in a subsequent paper.

\section{REFERENCES}

1. M. Auslander and O. Goldman, The Brauer group of a commutative ring, Trans. Amer. Math. Soc. 97 (1960), 367-409. MR 22 \#12130.

2. H. Bass, Algebraic K-theory, Benjamin, New York, 1968. MR 40 \#2736.

3. - Topics in algebraic K-theory, Lecture Notes, Tata Institute, Bombay, 1967.

4. S. U. Chase, D. K. Harrison and A. Rosenberg, Galois theory and Galois cohomology of commutative rings, Mem. Amer. Math. Soc. No. 52 (1965). MR 33 \#4118.

5. G. Janusz, Separable algebras over commutative rings, Thesis, University of Oregon, Eugene, Ore., 1965.

6. M. Karoubi, Fondements de la K-theorie, Faculté des Sciences de l'Université d'Alger, 1966/67.

7. J.-P. Sérre, Applications algébriques de la cohomologie des groupes: Théorie des algèbres simples, Séminaire H. Cartan 1950/51, exposés 5-7, Secrétariat mathématique, Paris, 1955. MR 17, 1117.

8. Corps locaux, Actualités Sci. Indust., no. 1296, Hermann, Paris, 1962. MR 27 \#133.

9. C. T. C. Wall, Graded Brauer groups, J. Reine Angew. Math. 213 (1963/64), 187-199. MR 29 \#4771.

Columbia University, New YoRK, New YoRK 10027

The City College of the City University of New York, New York, New York 10031 